\title{
Conformations and dynamics of stars and dendrimers: the Gaussian Self-Consistent approach
}

\author{
F.Ganazzoli* \\ Dipartimento di Chimica, Materiali e Ingegneria Chimica 'G. Natta' \\ Politecnico di Milano \\ via L.Mancinelli 7, 20131 Milano, Italy
}

Received June 1, 2001

Some conformational and dynamical aspects of branched polymer are reviewed. We discuss the theoretical Gaussian Self-Consistent (GSC) approach proposed in our group and used to study the behaviour of regular star polymers and dendrimers in different solvent conditions. Within a single framework, we consider the unperturbed $\Theta$ state, as well as the goodsolvent state in comparison with other theoretical or simulation approaches, and with some experimental results. We also briefly report the further results obtained for amphiphilic copolymer stars in selective solvents so as to highlight the potentialities of the method, as well as its strengths and its shortcomings.

Key words: star polymers, dendrimers, statistical mechanics, polymer conformations, polymer dynamics

PACS: $36.20 . E y, 61.25 . \mathrm{Hq}, 82.35 . \mathrm{Jk}, 82.35 . \mathrm{Lr}, 83.80 . \mathrm{Rs}$

\section{Contents}

$\begin{array}{llr}1 & \text { Introduction } & 38\end{array}$

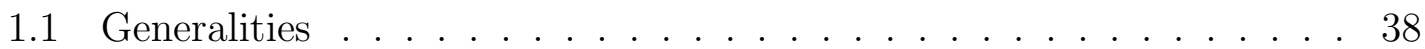

1.2 Stars and dendrimers . . . . . . . . . . . . . . . . . . 39

2 Mathematical background $\quad 41$

2.1 The intramolecular free energy and the intramolecular conformation . 41

2.2 The intramolecular dynamics: Zimm's approach . . . . . . . . . . . 43

3 The unperturbed state of stars and dendrimers $\quad 46$

3.1 The $\Theta$ temperature and the second virial coefficient . . . . . . . . . . 46

3.2 The molecular dimensions at the $\Theta$ temperature . . . . . . . . . . 50

*E-mail: fabio.ganazzoli@polimi.it 
4 The good-solvent expansion of stars and dendrimers 52

4.1 The molecular size and the local conformation . . . . . . . . . . . 52

4.2 The intramolecular dynamics in a good solvent . . . . . . . . . . 55

\section{Star polymers in a poor solvent and amphiphilic block-copolymer} stars

6 Concluding remarks

\section{Introduction}

\subsection{Generalities}

In recent years, branched polymers have been synthesized with an unprecedented control over molecular architecture [1-4]. Relevant examples are regular starbranched polymers, that have equal arms connected to a single multifunctional core, and dendrimers, that possess an exponentially increasing number of branch points with increasing molar mass. These synthetic achievements also revived the theoretical interest in such molecules, both because of their aesthetic appeal, and because of their practical importance, as witnessed by a number of reviews $[1,2,5,6]$. Moreover, these systems challenge at the same time fruitful theoretical approaches and long-held general paradigms, in particular about the unperturbed state. On the other hand, control over molecular architecture is astonishingly good, yet it is still not perfect. For instance, heavily branched stars often show an incomplete linking reaction, and analogous difficulties are met in growing sterically encumbered dendrimers [7]. This problem might be alleviated through different synthetic strategies, but it cannot be easily disposed of. For instance, it eventually limits the dendrimer growth to a finite, and usually relatively small, generation [8].

Many approaches were employed to study branched polymers, ranging from analytical renormalization group or scaling approaches to computer simulations. In our group, we proposed a molecular model to investigate both equilibrium and dynamics of linear and branched polymers in a unified approach based on a coarse-grained picture of the molecule. A basic assumption is the Gaussian distribution of the distances among the beads, where the interactions and the friction with solvent are concentrated [9]. The equilibrium conformation is obtained from self-consistent minimization of the intramolecular free energy, which provides the name of Gaussian Self-Consistent (GSC) approach. We correctly account for all the relevant degrees of freedom within the configurational entropy, and include two- and three-body interactions as the most representative many-body interactions. In dynamics, we adopt the stochastic Langevin equation taking into account the equilibrium results. We should mention that Timoshenko et al. have independently developed an equivalent version of the GSC approach to study the behaviour of homo- and copolymers in poor solvents [10].

In the next sections, we first describe regular star-branched polymers (or stars for brevity) and dendrimers, introducing some notation and a few results valid for the 
random-walk (RW) model and for the good-solvent state of linear polymers. After summarizing the mathematical background, we discuss the unperturbed state, with the $\Theta$-temperature depression of stars and dendrimers compared to linear chains, and their expansion compared to a random walk due to residual interactions. Afterwards, we consider the good-solvent state both through the molecular size and the intramolecular dynamics. Later on, we address the behaviour of amphiphilic star copolymers in selective solvents. The final section summarizes the main issues and provides an outlook for future work.

\subsection{Stars and dendrimers}

Regular stars are formed by $f$ equal arms emanating from a central core, each arm comprising $N / f$ beads connected by bond vectors of unit length for a total of $N$ bonds and $n_{\text {beads }}=N+1$ beads. Beads are sequentially numbered on each arm as shown in figure $1 \mathrm{a}$, where $f=5$ and $N / f=4$. Dendrimers are formed by $f$ trees, or arms, of order $m$ (the dendra) connected to a central core and comprising $P$ bonds between neighbouring branch points (see figure $1 \mathrm{~b}$, where $f=3, m=2$ and $P=1$ ). The topology of this class of molecules can be viewed as consisting of a sequence of concentric layers. The outmost layer defines the dendrimer generation $g$, numbered from 0 (no branch point beyond the central core) onward. The total number of beads is [11]

$$
n_{\text {beads }}=1+f \cdot P \cdot \frac{m^{g+1}-1}{m-1},
$$

which increases exponentially with the dendrimer generation $g$.

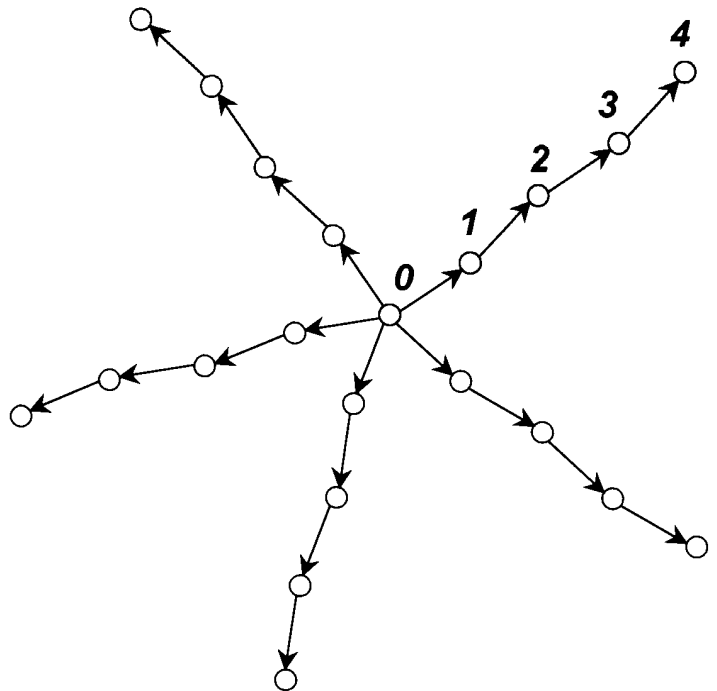

(a)

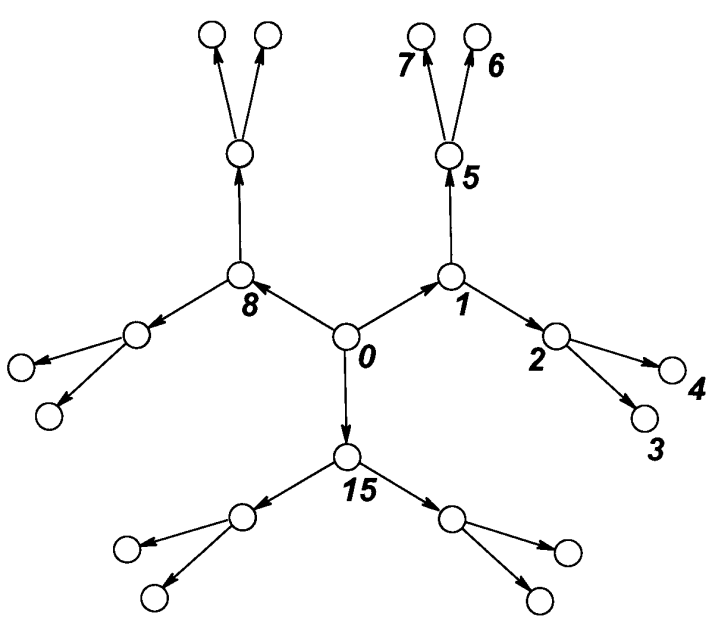

(b)

Figure 1. Schemes of regular stars (a) and dendrimers (b).

The molecular size is best discussed through the mean-square radius of gyration $\left\langle R_{\mathrm{S}}^{2}\right\rangle$. In linear and star polymers, its molar mass dependence is given by the power 
law $[12-17]$

$$
\left\langle R_{\mathrm{S}}^{2}\right\rangle \propto N^{2 \nu}
$$

where $\nu$ is the Flory exponent. For either topology, $\nu=0.5$ for the RW model and in the $\Theta$ state, and $\nu \cong 0.6$ in a good solvent (apart from $f$-dependent prefactors). Because of the common value of the exponent, it is also customary to introduce the topological ratio $g_{Q}=Q_{\text {star }} / Q_{\text {lin }}$ for molecules with the same molar mass in the same solvent conditions [5]. $Q$ may be $\left\langle R_{\mathrm{S}}^{2}\right\rangle$, the hydrodynamic radius $R_{\mathrm{H}}$, the intrinsic viscosity $[\eta]$, etc. In the limit of large molar mass, the RW model produces the simple result $[18]$

$$
g_{\mathrm{S}}^{\mathrm{RW}}=\frac{\left\langle R_{\mathrm{S}}^{2}\right\rangle_{\mathrm{star}}^{\mathrm{RW}}}{\left\langle R_{\mathrm{S}}^{2}\right\rangle_{\mathrm{lin}}^{\mathrm{RW}}}=\frac{3 f-2}{f^{2}},
$$

showing the obvious result that stars are more compact than linear chains with the same molar mass.

Conversely, dendrimers belong to a different universality class, as easily seen through the RW model. In fact, for dendrimers equation (1.2) is ruled out and the asymptotic result for $N \rightarrow \infty$, corresponding to $g \rightarrow \infty$, is given by $[11,19]$

$$
\left\langle R_{\mathrm{S}}^{2}\right\rangle=P(g+1)
$$

(in bond-length units). Thus, the radius of gyration is equal to the dendron span, as it is expected considering that one half of the beads lies on the outmost shell (see figure $1 b)$. In terms of the total number of bonds, we have

$$
\left\langle R_{\mathrm{S}}^{2}\right\rangle \propto \ln N
$$

Within the random-walk model, the Flory exponent $\nu=0.5$ of linear and star polymers corresponds to a fractal dimension $D=\nu^{-1}=2$. Conversely, in dendrimers $\nu$ is smaller than any non-zero value, since the logarithm increases more slowly than any power of its argument, hence the fractal dimension diverges. Interestingly, ideal randomly branched (cascade) molecules do still follow equation (1.2) with a Flory exponent that can be as low as $0.25[18,20]$, hence with a fractal dimension $D=4$. Therefore, dendrimers form an extreme case of regularly branched systems. Note that according to equation (1.2) the average density within a molecule is $d \propto$ $N /\left\langle R_{\mathrm{S}}^{2}\right\rangle^{3 / 2} \propto N^{1-3 \nu}$. The exponent $\nu=1 / 3$ corresponds to a density independent from molar mass, while for $\nu>1 / 3$ the density goes to zero with an increasing molar mass, and for $\nu<1 / 3$ it diverges. The latter situation is met with cascade molecules, and a fortiori with dendrimers because of equation (1.5). In conclusion, the RW model can never apply to such molecules [20], and dendrimers cannot exceed some definite molar mass so as to keep $d$ finite.

The relationship between radius of gyration and molar mass is further complicated in dendrimers by the finite span of the arms which prevents reaching any asymptotic behaviour. Therefore, we only find apparent $N$-dependent $\nu$ exponents (see later), and the $g_{S}$ ratio is meaningless for these systems. 


\section{Mathematical background}

\subsection{The intramolecular free energy and the intramolecular conformation}

In our statistical approach, the average scalar products among the bond vectors can be used to describe the chain conformation. We collect them in the symmetrical matrix M, with elements $[9,19,21,22]$

$$
M_{i j}=\left\langle\mathbf{l}_{i} \cdot \mathbf{l}_{j}\right\rangle .
$$

Knowledge of this matrix under the specific thermodynamic conditions affords all the mean-square equilibrium averages. For instance, the mean-square distances among the beads are given by the general expression [11]

$$
\left\langle r_{i j}^{2}\right\rangle=\sum_{u=1}^{D_{i j}} \sum_{v=1}^{D_{i j}} U(u, v)\left\langle\mathbf{l}_{b(u)} \cdot \mathbf{l}_{b(v)}\right\rangle,
$$

where the bond index $b(u)$ identifies the $u$-th step of the shortest path connecting beads $i$ and $j$, while $D_{i j}$ is the topological distance between the two beads and $U(u, v)= \pm 1$ accounts for the vector directions in the path (see figure 1 ). This equation simplifies for linear and star polymers, but no simple, yet general, expression can be written for dendrimers.

Taking the RW model as the reference state, the excess free energy of the single molecule in $k_{\mathrm{B}} T$ units is [9] $\mathcal{A}=\mathcal{A}_{\text {el }}+\mathcal{A}_{\text {intra }}$. $\mathcal{A}_{\text {el }}$ accounts for the configurational entropy, which determines the molecular elasticity (whence its name). Assuming a Gaussian distribution of the bond vectors, it is given by $[19,21,22]$

$$
\mathcal{A}_{\mathrm{el}}=\frac{3}{2}[\operatorname{Tr} \mathbf{M}-N-\ln \operatorname{Det} \mathbf{M}]=-\frac{3}{2} \ln \operatorname{Det} \mathbf{M}
$$

due to the equality $\operatorname{Tr} \mathbf{M}=\sum_{i=1}^{N}\left\langle\left|\mathbf{l}_{i}\right|^{2}\right\rangle=N$, which holds for constant (unit) bond lengths.

If short-range stereochemical interactions are ignored, $\mathcal{A}_{\text {intra }}$ is only due to topologically long-ranged intramolecular two- and three-body interactions. We express them as sums over all the distinct pairs and triplets of beads

$$
\mathcal{A}_{\text {intra }}=\mathcal{A}_{2}+\mathcal{A}_{3}=\beta_{2} \sum_{i<j} p_{i j}+\beta_{3} \sum_{i<j<k} p_{i j k},
$$

where $\beta_{2}$ and $\beta_{3}$ are the irreducible binary and ternary cluster integrals of the beads, while $p_{i j}$ and $p_{i j k}$ are the corresponding probability densities of contact. Under the assumption of a Gaussian distribution of the intramolecular distances we have: $[9,21,22]$

$$
p_{i j}=\left[\frac{3}{2 \pi\left\langle r_{i j}^{2}\right\rangle}\right]^{3 / 2},
$$




$$
\begin{gathered}
p_{i j k}=\left[\frac{3}{2 \pi}\right]^{3} \Psi_{i j k}^{-3 / 2} \\
\Psi_{i j k}=\frac{1}{2}\left[\left\langle r_{i j}^{2}\right\rangle\left\langle r_{i k}^{2}\right\rangle+\left\langle r_{i j}^{2}\right\rangle\left\langle r_{j k}^{2}\right\rangle+\left\langle r_{i k}^{2}\right\rangle\left\langle r_{j k}^{2}\right\rangle\right]-\frac{1}{4}\left[\left\langle r_{i j}^{2}\right\rangle^{2}+\left\langle r_{i k}^{2}\right\rangle^{2}+\left\langle r_{j k}^{2}\right\rangle^{2}\right] .
\end{gathered}
$$

We point out that only in special cases does $p_{i j k}$ factor out in the product $p_{i j} \cdot p_{j k}$, for instance in the RW model, and that the positivity requirement for $\Psi_{i j k}$ is related with the requirement of intramolecular connectivity [9].

Using equations (2.5) and (2.6), we then write

$$
\mathcal{A}_{\text {intra }}=b_{2} \sum_{i<j}\left\langle r_{i j}^{2}\right\rangle^{-3 / 2}+b_{3} \sum_{i<j<k} \Psi_{i j k}^{-3 / 2}
$$

with $b_{2}=\beta_{2} \cdot(3 / 2 \pi)^{3 / 2}$ and $b_{3}=\beta_{3} \cdot(3 / 2 \pi)^{3}$. The $b_{2}$ parameter depends on the solvent quality and is therefore temperature dependent. Thus, it can approximately be written as

$$
b_{2}=\tau B
$$

where $B$ is the adimensional covolume of a bead and

$$
\tau=\frac{T-\Theta_{\text {bead }}}{T} .
$$

Therefore, $\Theta_{\text {bead }}$ is the temperature that makes $\beta_{2}=0$. In a good solvent, the beads are well solvated, $b_{2}$ is positive and the two-body interactions are repulsive. Conversely, in a poor solvent $b_{2}$ is negative and the attractive interactions among the beads expel the solvent from the coil interior. The unperturbed $\Theta$ state, corresponding to vanishing interactions between two molecules, is more complicated, and does not correspond to the simple case $b_{2}=0$, i.e. $T=\Theta_{\text {bead }}$, when the two-body interactions among the beads vanish [14,20,23-25]. We discuss this case in section 3.

As to the $b_{3}$ parameter, it is always positive and essentially independent of temperature, so that these interactions are repulsive. In fact, they correct the excessive contribution of the independent two-body interactions involving three beads, because there is less room for the third bead to interact with the other two if these are already in contact. Note that the number of two- and three-body interactions are of the same order of magnitude, because each two-body interaction brings about a few three-body interactions involving a topologically neighbouring third bead due to molecular connectivity.

The chain conformation is obtained by minimizing the intramolecular free energy expressed through the scalar products $\left\langle\mathbf{l}_{i} \cdot \mathbf{l}_{j}\right\rangle$ that form our variational parameters. The problem becomes numerically intractable at large molar mass, since the number of variables is equal to $N(N-1) / 2$. By exploiting the molecular symmetry, we greatly reduce this number, but still we cannot study the asymptotic behaviour of stars. In this case, we choose a different strategy, consisting in optimizating the eigenvalues of $\mathbf{M}$ once we know its eigenvectors matrix $\mathbf{V}$ from symmetry considerations [9,24,26]. To this end, we collect the $N$ bond vectors of the star in the column vector $\mathbf{l}$, so that $\mathbf{l}^{T}=\left[\mathbf{l}_{1}, \mathbf{l}_{2}, \ldots, \mathbf{l}_{N}\right]$ and $\mathbf{M}=\left\langle\mathbf{1} \mathbf{l}^{T}\right\rangle$. Diagonalization of $\mathbf{M}$ can be carried out as 
discussed in [26]. The eigenvector matrix $\mathbf{V}$ transforms $\mathbf{l}$ into the orthogonal vector L:

$$
\begin{gathered}
\mathbf{L}=\mathbf{V} \cdot \mathbf{l}, \\
\left\langle\mathbf{L} \mathbf{L}^{T}\right\rangle=\mathbf{V}\left\langle\mathbf{l} \mathbf{l}^{T}\right\rangle \mathbf{V}^{T}=\mathbf{V} \cdot \mathbf{M} \cdot \mathbf{V}^{T}=\alpha^{2} .
\end{gathered}
$$

The elements of the eigenvalue matrix $\alpha^{2}$ are collected into two groups with different degeneracies, arising from the statistical symmetry among the star arms: $\alpha_{2 p}^{2}$, $p=1,2, \ldots, N / f$ with a unit multiplicity, and $\alpha_{2 p-1}^{2}, p=1,2, \ldots, N / f$ with an $f-1$ multiplicity, for a total of $N / f+(f-1) N / f=N$ eigenvalues, as it must be. Therefore, the elements of $\alpha^{2}$ are the strain ratios of the configurational normal modes with respect to the RW conformation where $\left\langle\mathbf{l} \mathbf{l}^{T}\right\rangle=\left\langle\mathbf{L} \mathbf{L}^{T}\right\rangle=\mathbf{1}$, i.e. the identity matrix. The statistically orthogonal elements of $\mathbf{L}$, the configurational normal modes, coincide with the dynamic normal modes in the free-draining regime (see also section 2.2). The mean-square distances and the configurational entropy are then written as sums over these normal modes [26], so that the intramolecular free energy depends only on the $2 N / f$ variational strain ratios $\alpha_{2 p}^{2}$ and $\alpha_{2 p-1}^{2}$.

There is however a problem with this choice of variables, which may be relevant in some cases. The elements of $\mathbf{V}$ are known a priori from symmetry considerations provided the molecule is conformationally homogeneous and the bond-vector correlation dies off quickly enough with topological separation. These requirements are not met in two important situations [21]: i) in copolymers, because of the presence of unlike monomers; ii) in a good solvent, because of long-range bond-vector correlation. In the former case, we must resort anyway to the full procedure [22], whereas in the latter case the normal-modes can still be satisfactorily used even with a large expansion, provided we are in the crossover regime. In fact, with the approximate form of $\mathbf{V}$, the diagonal terms of the $\alpha^{2}$ matrix are anyway close to the true eigenvalues of the star, and the neglected off-diagonal terms do not significantly affect the radius of gyration [26]. On the other hand, in dendrimers the bond-vector correlation is very large in all cases because of the molecular topology, and we must always adopt the complete procedure.

From the free energy minimization, we get the mean-square distances among the beads. Other quantities describing both the local and the overall conformation may be derived, in particular the mean-square radius of gyration

$$
\left\langle R_{\mathrm{S}}^{2}\right\rangle=\frac{1}{n_{\text {beads }}^{2}} \sum_{i<j}\left\langle r_{i j}^{2}\right\rangle
$$

and the mean-square distances of the generic $i$-th bead from the molecular center of mass

$$
\left\langle R_{i}^{2}\right\rangle=n_{\text {bead }}^{-1} \sum_{j}\left\langle r_{i j}^{2}\right\rangle-\left\langle R_{\mathrm{S}}^{2}\right\rangle
$$

\subsection{The intramolecular dynamics: Zimm's approach}

The dynamics is studied following Zimm's approach [27] with preaveraged hydrodynamic interaction, using a bead-and-spring model, each "bond" being replaced 
by a harmonic spring. The stochastic Langevin equation is written as $[11,19,28]$

$$
\frac{3 k_{\mathrm{B}} T}{l^{2}} \mathbf{H} \cdot \mathbf{A} \cdot \mathbf{R}+\zeta \dot{\mathbf{R}}=\mathbf{F},
$$

where $\mathbf{R}$ is the column vector formed by the the vector positions of the beads, $\zeta$ is their friction coefficient and $3 k_{\mathrm{B}} T / l^{2}$ is the spring constant. $\mathbf{F}$ is the vector containing the Brownian forces acting on the beads: each element of $\mathbf{F}$ has a zero mean and a non-zero second moment obtained through the fluctuation-dissipation theorem. The force-constant matrix $\mathbf{A}$ also depends on the molecular topology and is obtained by inverting $\mathbf{M}[29]$ :

$$
\mathrm{A}=\mathrm{G} \cdot \mathrm{M}^{-1} \cdot \mathrm{G}^{\mathrm{T}}
$$

Here, $\mathbf{G}$ is the incidence matrix which depends only on molecular topology [11]. The hydrodynamic interaction is embodied in matrix $\mathbf{H}$ with elements $[27,30,31]$ :

$$
H_{i j}=\delta_{i j}+\left(1-\delta_{i j}\right) \zeta_{r}\left\langle\frac{l}{r_{i j}}\right\rangle,
$$

where $\zeta_{r}=\zeta / 6 \pi \eta_{0} l$ is the reduced friction coefficient (set equal to $0.25[32]$ ), $\eta_{0}$ is the solvent viscosity and, in the Gaussian approximation, $\left\langle r_{i j}^{-1}\right\rangle=\left(6 / \pi\left\langle r_{i j}^{2}\right\rangle\right)^{1 / 2}$.

The dynamical problem reduces to solving the eigenvalue equation

$$
[\mathbf{H} \cdot \mathbf{A}] \cdot \mathbf{Q}=\mathbf{Q} \cdot \Lambda, \quad \Lambda=\left\{\lambda_{p} \delta_{p q}\right\}
$$

The diagonalization of the non-symmetrical matrix $\mathbf{H} \cdot \mathbf{A}$ can be performed numerically. The eigenvalue matrix $\Lambda$ contains a zero eigenvalue related to the diffusion of the center of mass, and the relaxation rates of the normal modes of motion $\lambda_{p}$, $p=1,2, \ldots, N$, which produce the viscoelastic relaxation times $\tau_{p}=\left(2 \sigma \lambda_{p}\right)^{-1}, \sigma=$ $3 k_{\mathrm{B}} T / \zeta l^{2}$ being the time unit. The eigenvectors, collected in matrix $\mathbf{Q}$, connect the column vector $\xi$ of the normal coordinates $\xi_{p}, p=0,1,2, \ldots, N$, to the column vector $\mathbf{R}$ of the vector positions of the beads $\mathbf{r}_{i}$ :

$$
\mathbf{R}=\mathbf{Q} \cdot \xi
$$

According to the Langevin equation, the autocorrelation function of the normal coordinates decays exponentially with time constants equal to $2 \tau_{p}$.

While the above procedure is fully general, using the bond vectors as the dynamic variables allows us a more efficient treatment of the molecular symmetry of stars. Since these variables are given by $\mathbf{l}_{i}=\mathbf{r}_{i}-\mathbf{r}_{i-1}$, after collecting the bond vectors in the row vector $\mathbf{l}$, the alternative equation reads [28,33]

$$
\frac{3 k_{\mathrm{B}} T}{l^{2}} \mathbf{B} \cdot \mathbf{M}^{-\mathbf{1}} \cdot \mathbf{l}+\zeta \mathbf{i}=\mathbf{F}^{\prime}
$$

where $\mathbf{B}=\mathbf{G}^{\mathbf{T}} \cdot \mathbf{H} \cdot \mathbf{G}$ accounts for the (preaveraged) hydrodynamic interaction. 
The spectrum of the relaxation times yields the dynamic viscosity, measured under an oscillating shear deformation with frequency $\omega[27,30]$

$$
[\eta(\omega)]=\lim _{c \rightarrow 0} \frac{\eta(\omega)-\eta_{0}}{c \eta_{0}}
$$

where $c$ is the polymer mass concentration. From the dynamic equations, we get

$$
\eta(\omega)=\eta_{0}+\frac{c R T}{M} \sum_{p=1}^{N} \frac{\tau_{p}}{1+\mathrm{i} \omega \tau_{p}}
$$

( $M$ being the molar mass) which yields the usual viscosity in the limit $\omega \rightarrow 0$. The complex modulus is obtained as $G^{*}(\omega)=G^{\prime}(\omega)+\mathrm{i} G^{\prime \prime}(\omega)=\mathrm{i} \omega \eta(\omega)$, i being the imaginary unit. Also, we get the oscillatory flow birefringence $S(\omega)$ :

$$
S(\omega)=S_{0}+\frac{2 c N_{\mathrm{Av}} \Delta \gamma}{3 M} \sum_{p=1}^{N} \alpha_{p}^{2} \frac{\tau_{p}}{1+\mathrm{i} \omega \tau_{p}},
$$

where $S_{0}$ is the solvent contribution, while $N_{\mathrm{Av}}$ is Avogadro's constant and $\Delta \gamma$ is the difference in polarizability along the bond axis and perpendicular to it. Both $\eta(\omega)$ and $S(\omega)$ can also be reported in terms of their modulus and phase angle as $\eta(\omega)=\eta_{M} \exp \left(-\mathrm{i} \varphi_{\eta}\right)$ and $S(\omega)=S_{M} \exp \left(-\mathrm{i} \varphi_{\mathrm{S}}\right)$. It is current practice to report the complex modulus through its real and imaginary components, and $S(\omega)$ in the phasor notation.

The relaxation times obtained from the eigenvalues of $\mathbf{H} \cdot \mathbf{A}$ or of $\mathbf{B} \cdot \mathbf{M}^{-\mathbf{1}}$ are often approximately expressed in linear chains through the power law $[9,27,30]$

$$
\tau_{p} \propto(N / p)^{\beta}
$$

or equivalently $\tau_{p}=\tau_{1} / p^{\beta}$ where $\tau_{1} \propto N^{\beta}$ is the longest relaxation time. The exponent is given by $\beta=3 \nu$, which yields $3 / 2$ in the $\Theta$ state and for the RW model, and $9 / 5$ in a good solvent $[9,30]$. From this relationship and equation $(2.22)$, we get the power-law dependence of the polymer contribution to the complex modulus

$$
G^{\prime}(\omega) \propto G^{\prime \prime}(\omega)-\omega \eta_{0} \propto \omega^{1 / \beta}
$$

when probing the intramolecular dynamics.

We also get the dynamic structure factor $S(q, t)$, measured in quasi-elastic scattering experiments. Here, $q$ is the modulus of the scattering vector given by $q=|\mathbf{q}|=$ $4 \pi \sin (\vartheta / 2) / \lambda, \vartheta$ being the scattering angle and $\lambda$ being the radiation wavelength. $S(q, t)$ is given by $[9,30]$ :

$$
S(q, t)=n_{\text {beads }}^{-2} \sum_{j, k}\left\langle\exp \left\{-\mathrm{iq} \cdot\left[\mathbf{r}_{k}(t)-\mathbf{r}_{j}(0)\right]\right\}\right\rangle .
$$

In the Gaussian approximation, we get

$$
S(q, t)=\exp \left[-q^{2} D t\right] \frac{1}{n_{\text {beads }}^{2}} \sum_{i \neq j} \exp \left[-\frac{q^{2}}{6}\left\langle r_{i j}^{2}(t)\right\rangle\right] .
$$


Here, we separated the contribution of the zero-th mode, yielding the diffusion coefficient $D$, from that of the internal modes, which produce the time-dependent mean-square distances $\left\langle r_{i j}^{2}(t)\right\rangle=\left\langle\left[\mathbf{r}_{j}(t)-\mathbf{r}_{i}(0)\right]^{2}\right\rangle-6 D t$ irrespective of the position of the center of mass. In bond-length units we have:

$$
\left\langle r_{i j}^{2}(t)\right\rangle=\left\langle r_{i j}^{2}\right\rangle+2 \sum_{p=1}^{N} Q_{i p} Q_{j p} \mu_{p}^{-1}\left[1-\exp \left(-t / 2 \tau_{p}\right)\right]
$$

where $\mu_{p}$ is the generic element of the diagonal matrix $\mathbf{Q}^{\mathbf{T}} \cdot \mathbf{A} \cdot \mathbf{Q}$. Note that for $t \rightarrow 0, S(q, t)$ reduces to the static structure factor, or form factor.

The dynamic structure factor is often characterized through its first cumulant $\Omega(q)$, produced by the initial logarithmic slope $[31,34]$

$$
\Omega(q)=-\left.\frac{\partial}{\partial t} \ln \left(\frac{S(q, t)}{S(q, 0)}\right)\right|_{t \rightarrow 0} .
$$

In principle, $\Omega(q)$ is somewhat ill-defined because of the $t \rightarrow 0$ limit due to the local relaxation within a monomer. While this problem does not arise when using coarse-grained models, the experimental time scales of local and of collective dynamics usually differ by a few orders of magnitude, so that in practice they are easily separated in appropriate experiments. This may not be true in the case of stiff polymers, where there is no sharp distinction between local and collective time scales.

Neglecting such an issue, the first cumulant can be calculated as [34]

$$
\Omega(q)=\frac{q^{2} l^{2} \sigma}{S(q)}\left[1+\frac{\zeta_{\mathrm{r}}}{n_{\text {beads }}} \sum_{i, j}\left(1-\delta_{i j}\right)\left\langle\frac{l}{r_{i j}}\right\rangle f\left(x_{i j}\right)\right],
$$

$\left\langle l / r_{i j}\right\rangle$ being obtained from $\left\langle r_{i j}^{2}\right\rangle$ in the Gaussian approximation. $x_{i j}$ is the dimensionless variable $x_{i j}=q \cdot\left(\left\langle r_{i j}^{2}\right\rangle / 6\right)^{1 / 2}$, while $f(x)$ may be calculated with or without preaveraging the hydrodynamic interaction:

$$
f(x)= \begin{cases}\exp \left(-x^{2}\right) & \text { with preaveraging, } \\ \frac{3}{4}\left(x^{-3}+x^{-1}\right) \mathrm{e}^{-x^{2}} \int_{0}^{x} \mathrm{e}^{-t^{2}} \mathrm{~d} t-x^{-2} & \text { without preaveraging. }\end{cases}
$$

Therefore, computation of the first cumulant enables us to gauge the error entailed by this approximation.

\section{The unperturbed state of stars and dendrimers}

\subsection{The $\Theta$ temperature and the second virial coefficient}

The unperturbed state is experimentally achieved when the second virial coefficient $A_{2}$ vanishes for the given polymer/solvent pair, and the solution shows a 
(pseudo) ideal behaviour [12]. The temperature producing $A_{2}=0$ is the $\Theta$ temperature, analogous to the Boyle temperature of real gases. Generally, $\Theta$ is taken as independent from molar mass, as indeed observed in (long) linear chains. This is consistent with the notion of a vanishing binary cluster integral of the beads: attractive and repulsive interactions exactly cancel one another and the $\Theta$ state can be described in terms of non-interacting molecules with a RW conformation. This prediction, in excellent agreement with observation, leads to the notion of universality of the $\Theta$ state $[12,13,30]$.

In recent years, however, experimental results have shown that neither the $\Theta$ temperature of stars nor their $\Theta$ dimensions show any universality $[5,6,35]$. In particular, stars show an $f$-dependent depression of the $\Theta$ temperature compared to linear chains, although at large molar mass the same asymptotic value is reached. Moreover, even in this limit, stars have a larger size than expected for the RW model, and accordingly exhibit residual intramolecular interactions, unlike linear chains.

To explain this behaviour, we calculate the $\Theta$ temperature that makes $A_{2}=0$. This condition is met when the intermolecular free energy $\mathcal{A}_{\text {inter }}(1,2)$ vanishes $[13,23]$

$$
\mathcal{A}_{\text {inter }}(1,2)=\mathcal{A}(1,2)-\mathcal{A}(1)-\mathcal{A}(2)=0,
$$

$\mathcal{A}(1), \mathcal{A}(2), \mathcal{A}(1,2)$ being the free energies of the two molecules and of their ensemble. $\mathcal{A}_{\text {inter }}(1,2)$ is given by the two- and three-body interactions between two molecules:

$$
\mathcal{A}_{\text {inter }}(1,2)=n_{\text {beads }}^{2} \cdot \tau B+2 n_{\text {beads }} \cdot b_{3} \sum_{i<j}\left\langle r_{i j}^{2}\right\rangle^{-3 / 2}
$$

The first addendum accounts for the two-body interactions among the beads of the two molecules, and the second one for the three-body interactions among the $n_{\text {beads }}$ beads of the first molecule and all the $(i, j)$ pairs of the second molecule, the factor 2 taking into account the interchange between the molecules. Therefore, $\Theta$ is obtained from

$$
\tau_{\Theta} B=-2 \frac{b_{3}}{n_{\text {beads }}} \sum_{i<j}\left\langle r_{i j}^{2}\right\rangle^{-3 / 2},
$$

where

$$
\tau_{\Theta}=\left(\Theta-\Theta_{\text {bead }}\right) / \Theta
$$

Thus, $\Theta$ must be smaller than $\Theta_{\text {bead }}$ as an effect of molecular connectivity. Furthermore, in stars, the larger is the interaction multiplicity the larger are the three-body repulsions between two molecules, hence the larger must be the two-body attractions and thus the lowering of $\Theta[24,36]$.

Numerical calculation of $\Theta$ through equation (3.3) is coupled to the issue of the molecular conformation, because it requires the knowledge of the actual mean-square distances $\left\langle r_{i j}^{2}\right\rangle$. In turn, these can be obtained by self-consistent minimization of the intramolecular free energy at the current $\Theta$ temperature (i.e., with $b_{2}$ equal to the current $\left.\tau_{\Theta} B\right)[24,25]$.

Incidentally, for ring polymers equation (3.2) should also include an entropic term accounting for the loss of translational degrees of freedom due to the impossibility 


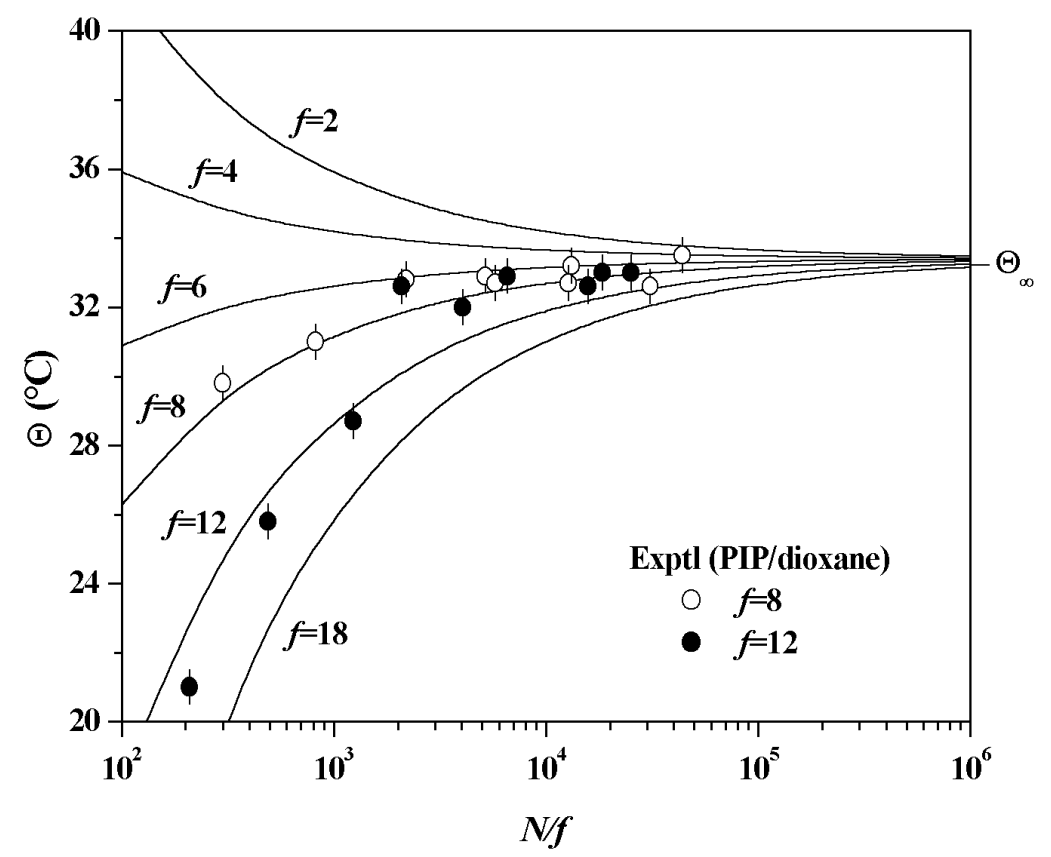

Figure 2. The $\Theta$ temperature of star polymers as a function of the number of skeletal atoms per arm $N / f$. The experimental results for 8- and 12-arm poly(isoprene) in dioxane are taken from [35]. For this system, the asymptotic $\Theta$ temperature is $\Theta_{\infty}=33.4^{\circ} \mathrm{C}$, while the fitting parameter is $\varphi=52.7 \mathrm{~K}$.

to have interlocked rings. Such a term effectively yields a repulsive potential that must be compensated by attractive two-body interactions, i.e., by a lowering of $\Theta$ compared to linear chains that is roughly independent of molar mass [37].

The results of our calculation indicate a striking difference between stars and dendrimers. In stars, $\Theta$ increases with the arm length $N / f$ (or equivalently with molar mass) to the same asymptotic limit $\Theta_{\infty}$ as in linear chains, as shown in figure 2, because in this limit the star topology becomes irrelevant. Moreover, at a fixed arm length, the $\Theta$ depression increases with the arm number $f$ as an effect of the larger multiplicity of intermolecular interactions. $\Theta$ changes with $N / f$ according to the square-root law:

$$
\Theta=\Theta_{\infty}-\varphi \cdot \chi_{f}(N / f)^{-1 / 2}
$$

showing that indeed $\Theta$ becomes asymptotically equal to $\Theta_{\infty}$ for both linear and star polymers if $N / f \rightarrow \infty$. In equation $(3.5) \chi_{f} \cong-2.628+0.5767 \cdot f-0.00988 \cdot f^{2}$, which is positive for $f>4$, so that $\Theta_{\infty}$ is reached from below (see also figure 2). Formally $\varphi=4 b_{3} \Theta_{\text {bead }} / B$, but it is best treated as an adjustable parameter. Here both $\varphi$ and $\chi_{f}$ are obtained from full analysis of the problem unlike what was done in [23], no recourse being made to the RW model.

On the other hand, in dendrimers, $\Theta$ decreases without limit with increasing generation $g$, as shown in figure 3 for the dendrimers of figure $1 \mathrm{~b}$, although only finite, and usually small, $g$ values can be realized in practice because of the increasingly larger monomer density. The reason for this behaviour is apparent: increasing $g$ 


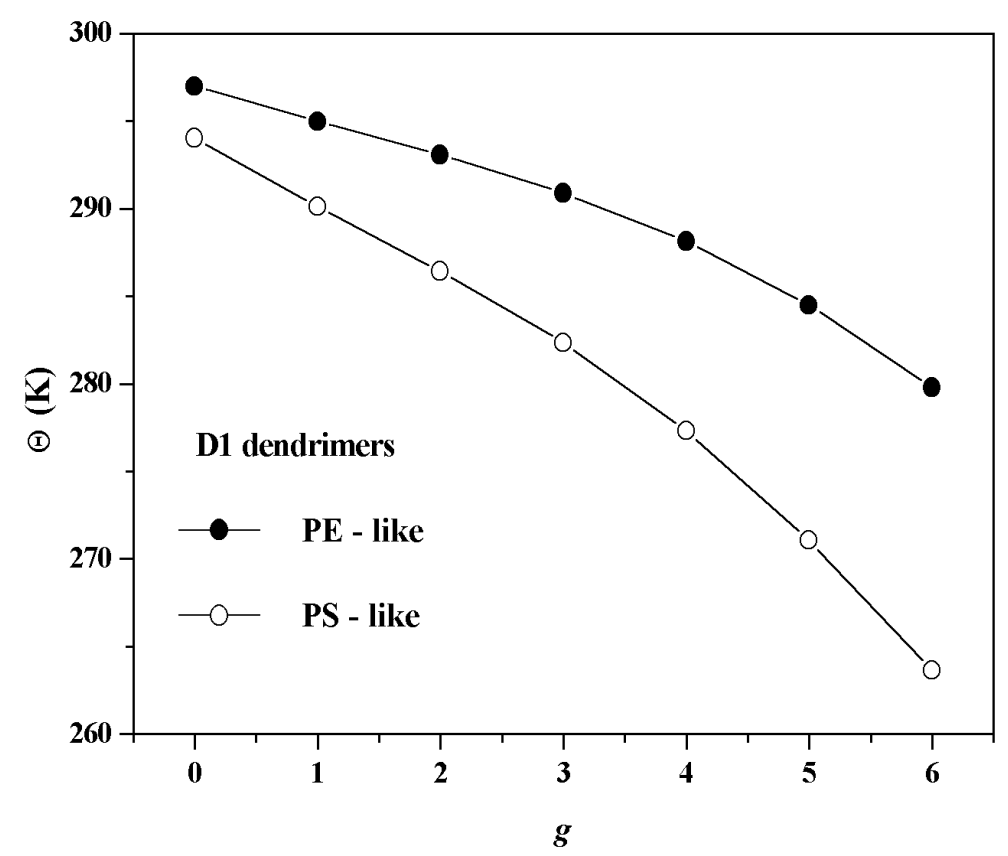

Figure 3. The calculated $\Theta$ temperature of the dendrimers shown in figure 1b. Here we arbitrarily chose $\Theta_{\text {bead }}=300 \mathrm{~K}$, and an adimensional covolume per bead $B=0.1$. The three-body interaction parameter was taken as $b_{3}=2 \cdot 10^{-3}$ for PS-like and $b_{3}=1 \cdot 10^{-3}$ for PE-like dendrimers, according to the values obtained for the corresponding star polymers (see figure 4).

also brings about a larger interaction multiplicity, so that from this viewpoint a dendrimer with an increasing $g$ can be compared to a star with a constant arm length and a larger number of arms. The $\Theta$ temperatures of figure 3 were obtained after choosing tentative, though realistic, values for the parameters $b_{3}, \Theta_{\text {bead }}$ and $B$ in view of the lack of experimental data [25]. The $B$ value corresponds to a relatively slim bond, while for $b_{3}$ we used the values yielding the correct radii of gyration for polyethylene and polystyrene stars in the $\Theta$ state (see next section).

The opposite trend of $\Theta$ that was just described is also obtained semiquantitatively from a very simple, smoothed density model similar to that employed in [38]. Let us assume in equation (3.3) that the density probability of contact between two beads, proportional to $\left\langle r_{i j}^{2}\right\rangle^{-3 / 2}$ in the GSC approach, be simply set equal to $V^{-1}$, where $V$ is the volume pervaded by the molecule. With this replacement, we have

$$
\tau_{\Theta} B \propto-\frac{1}{n_{\text {beads }}} \cdot \frac{n_{\text {beads }}^{2}}{V}=-\frac{n_{\text {beads }}}{V}=-d .
$$

From the discussion at the end of section 1.2, we conclude that in linear and star polymers $\tau_{\Theta}=\left(\Theta-\Theta_{\text {bead }}\right) / \Theta$ tends to zero with increasing molar mass just as its average density, and therefore $\Theta$ tends to the finite limit $\Theta_{\text {bead }}$ (instead of $\Theta_{\infty}$ ) from below. The correspondence with the full result is even better, considering that $V \propto\left\langle R_{\mathrm{S}}^{2}\right\rangle^{3 / 2} \propto\left(g_{\mathrm{S}} N\right)^{3 / 2}$. Using the RW result of equation (1.3) at large $f$, so that $g_{\mathrm{S}} \propto f^{-1}$, we get $\tau_{\Theta} \propto-f \cdot(N / f)^{-1 / 2}$. This result yields the same $\Theta$ dependence 


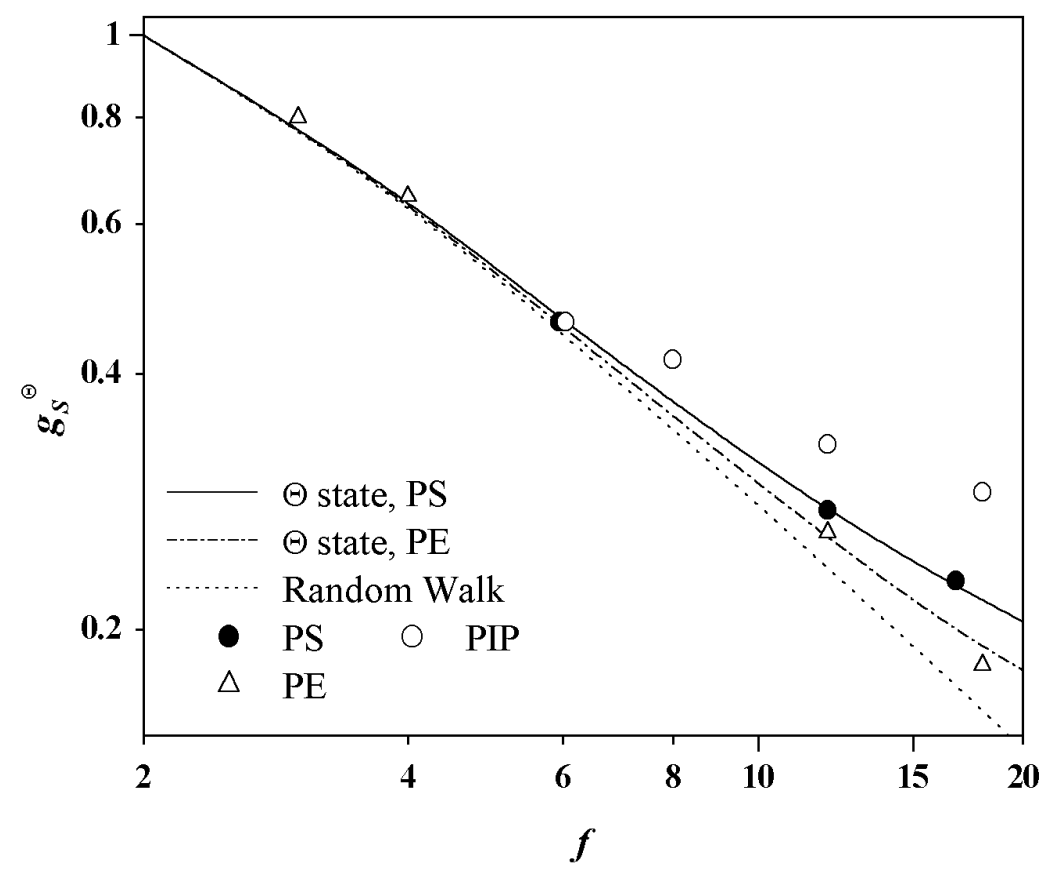

Figure 4. The topological ratio of the mean-square radii of gyration of stars in the $\Theta$ state $g_{\mathrm{S}}^{\Theta}$ plotted as a function of the number of arms $f$. The dotted line shows the random-walk prediction $g_{\mathrm{S}}^{\mathrm{RW}}=(3 f-2) / f^{2}$, the solid and the dashand-dot line the calculated results for PS and PE. The experimental results are taken from $[35,42]$ (PIP), [39] (PS) and [40,41] (PE).

from $N$ and $f$ as the full treatment with the RW model [the actual dependence is stronger, see equation (3.5)]. Conversely, in cascade polymers, and a fortiori in dendrimers, $d$ diverges and therefore $\tau_{\Theta}$ tends to $-\infty$ for $g \rightarrow \infty$, so that $\Theta$ decreases without limit, in agreement with figure 3.

\subsection{The molecular dimensions at the $\Theta$ temperature}

In linear chains, the attractive two-body interactions due to the depression of $\Theta$ compared to $\Theta_{\text {bead }}$ compensate almost exactly the intramolecular three-body repulsions. Therefore, linear chains can be described through an equivalent RW model. This is not so in stars and in dendrimers, where residual three-body repulsions involving three beads on three different arms or dendra are inherently non-compensated, lacking any intermolecular counterpart. These residual interactions give rise to a finite molecular swelling $[5,24]$ that can be described through the expansion factor $\alpha_{\mathrm{S}}^{2}$ of the mean-square radius of gyration with respect to the RW value,

$$
\alpha_{\mathrm{S}}^{2}=\left\langle R_{\mathrm{S}}^{2}\right\rangle /\left\langle R_{\mathrm{S}}^{2}\right\rangle^{\mathrm{RW}}
$$

In stars, the topological ratio $g_{\mathrm{S}}$ can be written in terms of $\alpha_{\mathrm{S}}^{2}$ :

$$
g_{\mathrm{S}}=g_{\mathrm{S}}^{\mathrm{RW}} \cdot \alpha_{\mathrm{S}, \text { star }}^{2} / \alpha_{\mathrm{S}, \text { lin }}^{2}
$$




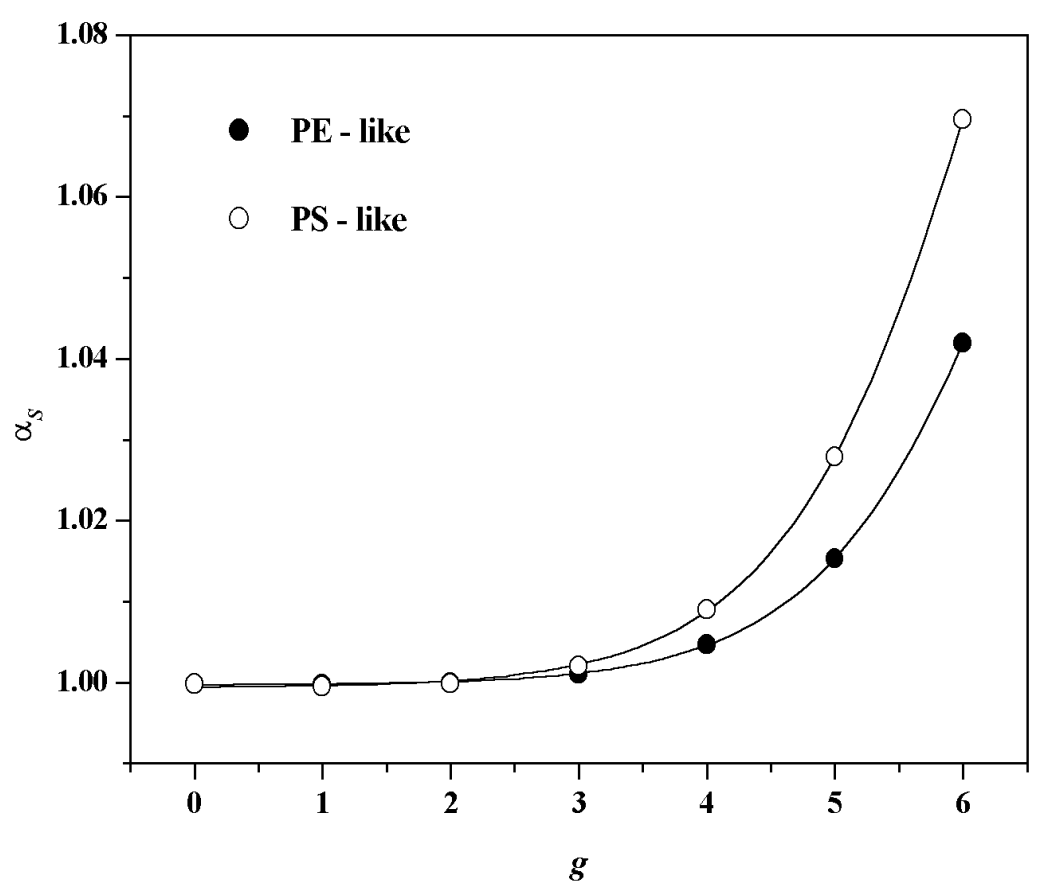

Figure 5. The $\Theta$-state expansion factor of the radius of gyration for dendrimers of figure $1 \mathrm{~b}$ plotted as a function of their generation $g$. The calculations for PSlike and PE-like dendrimers were carried out with the same $b_{3}$ parameters as in figures 2 and 3. More details on the fitting curve are reported in [25].

so that we get $g_{\mathrm{S}}^{\Theta}>g_{\mathrm{S}}^{\mathrm{RW}}$, since $\alpha_{S \text {, lin }}$ is equal to unity to a very good approximation. The $g_{\mathrm{S}}^{\Theta}$ ratio for regular stars is reported in figure 4. Here we compare our results, valid for atactic polystyrene (PS) and polyethylene (PE), with the RW prediction and with experimental data from PS [39], PE [40,41] and poly(isoprene) (PIP) [35, 42], showing the non-universality of the $\Theta$ state [5]. The PE values were tentatively obtained through equations (3.7) and (3.8) by comparison of the radii of gyration measured in solution and in the melt. Indeed, we argued that the RW conformation is achieved in a melt because of the screening of all interactions exerted by the neighbouring molecules, unlike what happens in the $\Theta$ state [40]. The agreement of our calculation with the experimental results is excellent, considering also that the $b_{3}$ parameters were previously determined for PS from an independent fit with entirely different experimental results [24], and for PE from a theoretical estimate by scaling the PS parameter through the monomer covolume and the characteristic ratios of the two polymers [40]. Interestingly, the simple scaling approach [43] predicts $g_{\mathrm{S}} \sim$ $f^{-1 / 2}$. This power law disagrees with experimental results, as well as with the nonuniversality of $g_{\mathrm{S}}^{\Theta}$. In our view, the basic assumption made in such an approach of a melt-like RW conformation near the star core is hard to reconcile with the topological constraint of the branch point.

In dendrimers, the multiplicity of three-body interactions increases with generation, leading to a sharp increase of molecular size over the RW value, as shown in figure 5 through $\alpha_{\mathrm{S}}$ as a function of $g[25]$. The curves are best fitted by a sigmoidal 
function, consistent with the finite expansion eventually achieved by the molecules because of their connectivity, although the upper asymptote (not shown) is reached at a generation that is probably too large to be realistic. As far as we know, the only other approach to study the dendrimer size in the $\Theta$ state was carried out through computer simulations [44]. However, this work adopted the interaction potential of linear chains (i.e., the same $T$ ), which suggests that the dendrimers are progressively moving in the good-solvent regime with the increasing generation [25]. Therefore, these results should be viewed with caution.

In this connection, we point out that a potentially serious shortcoming of computer simulations lies in the criterion adopted for the $\Theta$ temperature, usually found by looking for the relationship $\left\langle R_{\mathrm{S}}^{2}\right\rangle \propto N$ to be obeyed for a single molecule (see for instance [17] and [45]). On the other hand, the thermodynamic definition of $\Theta$ in terms of a vanishing $A_{2}$ requires simulating a two-chain system, which is obviously much harder. Only the approaches accounting for intermolecular interactions, as done for instance in [46], can provide definite answers, but unfortunately are extremely lengthy with present computers.

As a conclusion of this section, we stress that the non universality of the $\Theta$ state is related with the non-universality of the three-body interactions parameter $b_{3}$. Since its value is proportional to the sixth power of the ratio between the "bond" length and the local thickness, slight differences among different polymers may significantly affect the $\Theta$ state.

\section{The good-solvent expansion of stars and dendrimers}

\subsection{The molecular size and the local conformation}

In a good solvent, the repulsive two-body interactions swell the molecules compared to the $\Theta$ state. The expansion differs qualitatively from that due to the residual interactions in the $\Theta$ state. Within the GSC approach, the molecular conformation can be obtained by self-consistent free energy minimization neglecting the threebody interactions, effectively absorbed in the renormalized two-body interactions ("dressed" interactions [47]). Simultaneous two-body interactions are formally treated as independent in a mean-field approach, although they indirectly influence one another.

The result is a non-affine molecular expansion that in linear chains is greater at the chain center because of the additional interactions involving the outer beads $[9,26]$. This non-affinity is more pronounced in stars, being concentrated near the branch-point. Here the arms are stretched outward so as to minimize at the same time both the intra- and the inter-arm repulsions with little correlation among the initial arm directions, the more so the larger is the core functionality [26]. These theoretical predictions were nicely confirmed by off-lattice Monte Carlo simulations [48]. On the other hand, they are at variance with the basic assumptions of the scaling approach, which depicts the central part of the molecule as a melt [43]. However, since our results were obtained for relatively lightly branched stars $(f \leqslant$ 


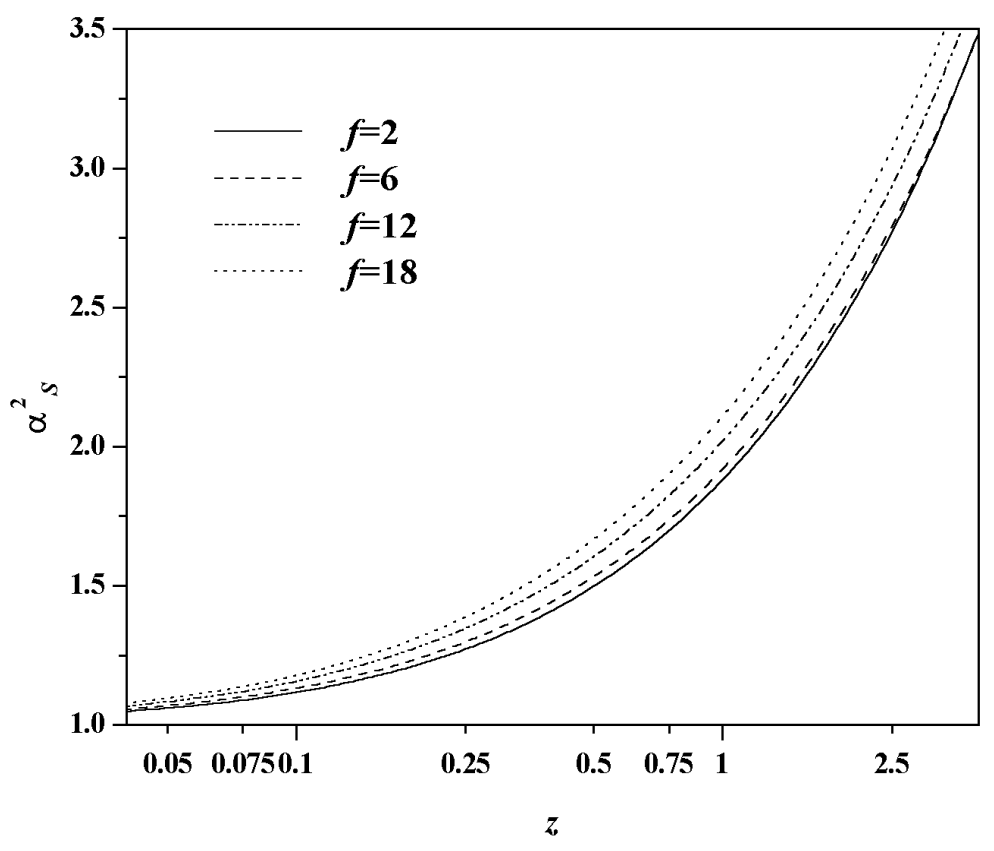

Figure 6. Universal plots of $\alpha_{\mathrm{S}}^{2}$ vs. the reduced variable $z=\tau B \sqrt{N}$ for linear $(f=2)$ and star $(f=6,12,18)$ polymers in a good solvent.

18), we speculate that scaling approaches are adequate for more heavily branched stars [6]. In dendrimers, the molecular expansion must account for the repulsive interactions both among the main dendra and among the sub-dendra, etc. This is achieved by an outward stretching of the individual dendra, sub-dendra, etc. with incipient dendron segregation and little inter-dendra mixing, in agreement with computer simulations [49]. Interestingly, we also find this effect to be enhanced in looser structures comprising two bonds between neighbouring branch points, which are kept apart as much as possible.

In spite of the non-affine expansion, linear and star polymers in a good solvent display a universal behaviour at a large molar mass when the appropriate reduced variables are adopted. This is achieved by plotting the expansion factor of the mean-square radius of gyration $\alpha_{\mathrm{S}}^{2}$ vs. $z=b_{2} \sqrt{N}$. In the large-expansion limit, the dependence is simply expressed through a power law

$$
\alpha_{\mathrm{S}} \propto z^{2 \nu-1}
$$

which yields equation (1.2) in view of the definitions of $\alpha_{\mathrm{S}}$ and of $z$. Conversely, at small $z$, the exact perturbative result is obtained:

$$
\alpha_{\mathrm{S}}^{2}=1+K_{f} z
$$

where the the analytical expression of $K_{f}$ is reported in [13]. $K_{f}$ slowly increases with $f$ above the value 134/105 of the linear chain, being eventually proportional to $f^{1 / 2}$.

Figure 6 reports the universal plots for $\alpha_{\mathrm{S}}^{2}$ obtained by the GSC approach through optimization of the approximate eigenvalues of $\mathbf{M}$ and neglecting the off-diagonal 
terms (see [26]). The numerical results in the cross-over region are fitted to a good approximation by the following expressions:

$$
\begin{array}{ll}
\alpha_{\mathrm{S}}^{2}=\left[1+9.571 z+5.81 z^{2}+106.6 z^{3}\right]^{2 / 15} & (f=2, \text { linear }), \\
\alpha_{\mathrm{S}}^{2}=\left[1+10.866 z+15.7 z^{2}+113.1 z^{3}\right]^{2 / 15} & (f=6), \\
\alpha_{\mathrm{S}}^{2}=\left[1+13.276 z+28.3 z^{2}+163.0 z^{3}\right]^{2 / 15} & (f=12), \\
\alpha_{\mathrm{S}}^{2}=\left[1+15.402 z+37.2 z^{2}+232.0 z^{3}\right]^{2 / 15} & (f=18) .
\end{array}
$$

In these equations, the exponent outside the square brackets was chosen so as to yield the Flory exponent $\nu=0.6$, while the linear term gives the correct perturbative results.

In linear and lightly branched star polymers, $\alpha_{\mathrm{S}}^{2}$ essentially follows the same function of the reduced variable $z$ due to the compensating effects of the arms expansion and of their increasing loss of correlation across the branch point. Accordingly, the topological ratio $g_{\mathrm{S}}^{\mathrm{GS}}=g_{\mathrm{S}}^{\mathrm{RW}} \cdot \alpha_{\mathrm{S} \text {, star }}^{2} / \alpha_{\mathrm{S} \text {, lin }}^{2}$ is very close to the RW value because of the cancellation of the effects within the expansion factors of linear and star polymers. An analogous result was previously obtained with the renormalization group approach [5]. In conclusion, remembering also the result of section 3.2 we have in general $g_{\mathrm{S}}^{\Theta}>g_{\mathrm{S}}^{\mathrm{GS}} \cong g_{\mathrm{S}}^{\mathrm{RW}}$.

Going back to the expansion factors of linear and star polymers, we note that a given $z=b_{2} \sqrt{N}$ for a fixed monomer/solvent pair, i.e., a fixed $b_{2}$, implies also the same molar mass. In stars with an increasing core functionality, this is achieved by decreasing the arm length. Conversely, in stars with the same arm length (the linear chain being the degenerate star with $f=2$ ), the molar mass increases linearly with the number of arms, hence $z$ is correspondingly larger by a factor $f^{1 / 2}$, with a larger molecular swelling.

Dendrimers in a good solvent follow a universal behaviour too, but because of their different fractal dimension a different reduced variable is required. Numerical results obtained with the GSC approach produce universal curves when plotted as a function of $\tau B N^{\varphi}$, with an empirical exponent $\varphi=0.923$ [19], as shown in figure 7 . Since for linear and star polymers the reduced variable is $\tau B \cdot N^{(4-d) / 2}[14], d$ being here the space dimension, the $\varphi$ exponent of dendrimers is significantly larger than the value $\varphi=0.5$ for $d=3$, but quite close to $\varphi=1$ for $d=2$ of linear and star polymers, consistent with the fact that a large fraction of the beads lies near the molecular surface. The dendrimer size is predicted to increase above the RW dimension up to a constant value with increasing generation and/or with increasing solvent quality. This asymptotically finite expansion is in sharp contrast to what is shown by stars, where the arm length can in principle increase without limit. This result is consistent with the finite maximum extension in real molecules, where the local stereochemistry is not affected by the solvent quality. Accordingly, for dendrimers we fitted a saturation Hill function to $\alpha_{\mathrm{S}}$ :

$$
\alpha_{\mathrm{S}} \cong 1+A_{\mathrm{H}} \frac{x^{\mathrm{H}}}{x_{1 / 2}^{\mathrm{H}}+x^{\mathrm{H}}},
$$




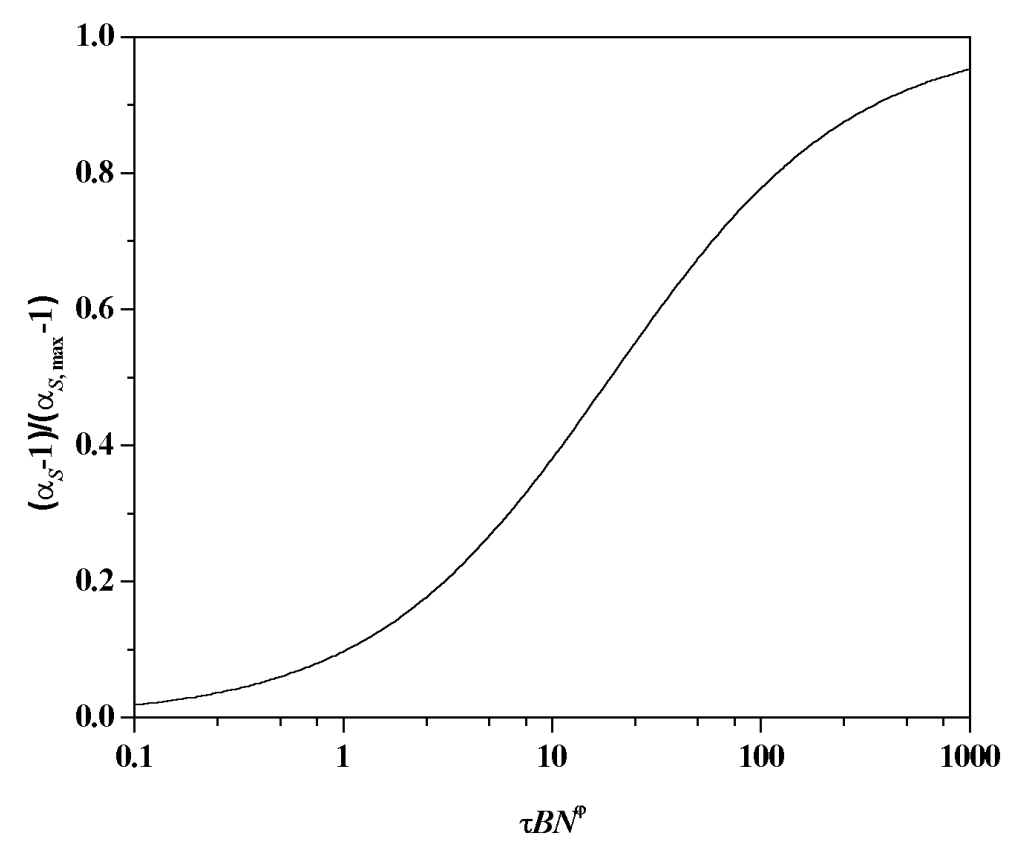

Figure 7. Universal plot of the expansion factor of the radius of gyration for the dendrimers of figure $1 \mathrm{~b}$ in a good solvent plotted as $\left(\alpha_{\mathrm{S}}-1\right) /\left(\alpha_{\mathrm{S}, \max }-1\right)$ vs. the reduced variable $\tau B N^{\varphi}$. Here $\varphi=0.923$, and $\alpha_{\mathrm{S}, \max }$ is the asymptotic maximum expansion of the molecule with fixed bond lengths [19], while the $H$ and $x_{1 / 2}$ parameters of equation (4.7) have the best-fit values $H=0.75$ and $x_{1 / 2}=20.33$.

where $x=\tau B N^{\varphi}$ is the reduced variable, while $A_{\mathrm{H}}, x_{1 / 2}$ and $H$ are the fitting parameters (see figure 7 ). Here $A_{\mathrm{H}}=\alpha_{\mathrm{S}_{\max }}-1$ is related to the strain ratio of the asymptotic maximum expansion of the molecule, while $\alpha_{\mathrm{S}}$ attains one half of the maximum increase above unity for $x=x_{1 / 2}$ [19]. Moreover, we also find that the topologically equivalent dendrimers with two bonds between adjacent branch points do follow the same curve, apart from a rigid shift along the logarithmic abscissa.

\subsection{The intramolecular dynamics in a good solvent}

A characteristic feature of regularly branched polymers consists in the degeneracy of the relaxation times. Since this degeneracy is due to the (average) molecular symmetry, it is present in all solvent conditions, regardless of the hydrodynamic interaction, although the exact ordering of the relaxation times might be affected. The star symmetry gives rise to even modes, related to the concerted motion of the arms, with a unit multiplicity, and to odd modes, describing the independent motion of the arms, with an $(f-1)$-multiplicity [33,50], i.e., the degeneracies of the eigenvalues of $\mathbf{M}$ discussed after equation (2.12). Because of the involved motion, the relaxation times of the odd modes follow the same power law of equation (2.24) as linear chains, unlike those of the even modes which however are of lesser importance. The complicated structure of dendrimers produces a whole hierarchy of relaxation times, whose general structure and multiplicity is reported in [31,51]. An example of this pattern is shown in figure 8 for the 6 th generation dendrimer of figure $1 \mathrm{~b}$. 


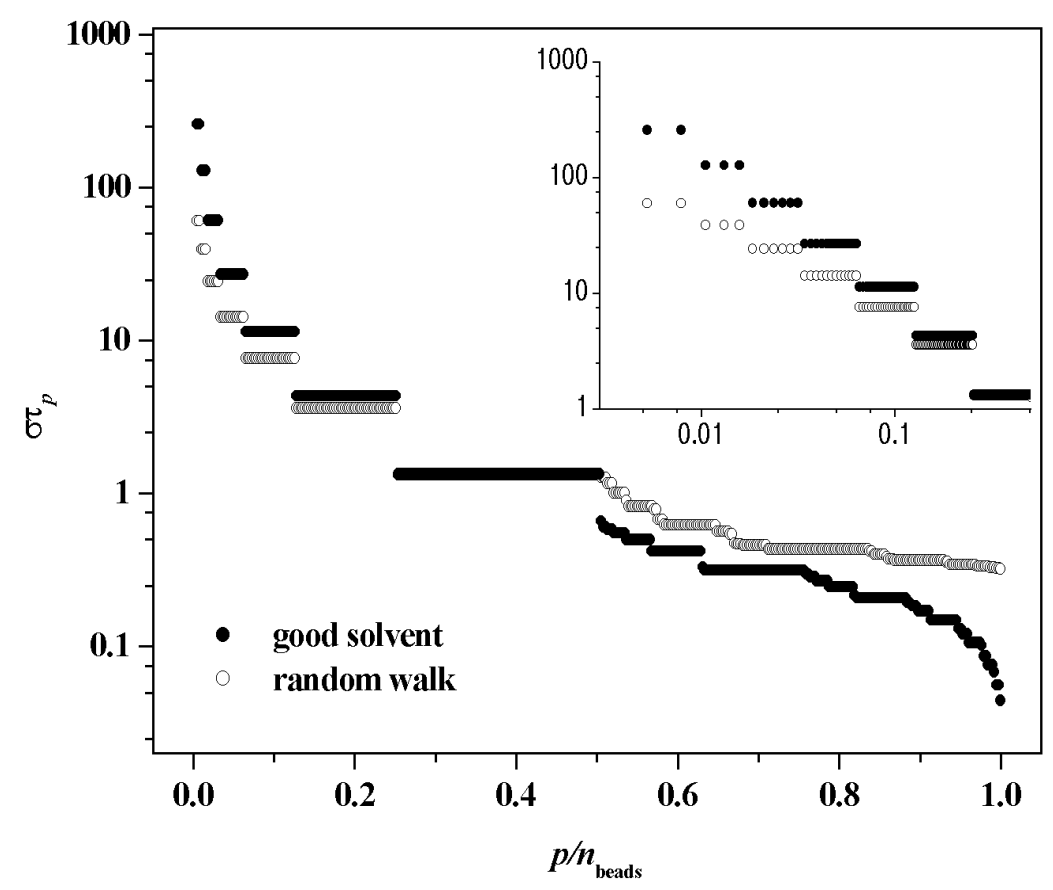

Figure 8. The relaxation times $\tau_{p}$, in $\sigma^{-1}$ units, of the 6 th generation dendrimer of figure $1 \mathrm{~b}$ as a function of the normalized mode index $p$. The inset shows an expanded view of the collective modes on a logarithmic abscissa.

As a general feature, the relaxation times become larger with molecular expansion, at least for the collective modes [see figure 8 and equation (2.24)], so that the instantaneous intramolecular conformation changes more slowly. This longer memory has a two-fold origin: i) the change in the chain elasticity embodied in the $\mathbf{A}$ matrix or equivalently in the $\mathbf{M}^{-\mathbf{1}}$ matrix [see equation (2.16)]; ii) the change in the hydrodynamic interaction, embodied in the $\mathbf{H}$ matrix [see equation (2.17)]. Because of that, in a good solvent, the intramolecular dynamics is moving toward the partial draining regime, intermediate between Zimm's impermeable coil limit and Rouse's free draining limit [33].

The dynamical eigenvalue problem in the presence of good-solvent expansion may be solved numerically. A useful and entirely adequate approximation for linear and star polymers consists in using the free-draining normal modes of the RW model, that are known analytically. Their use in the presence of hydrodynamic interaction (the so-called Zimm-Hearst approximation) entails a negligible error even with goodsolvent expansion for stars at least up to $f=18$ [33]: in particular, derived quantities such as the intrinsic viscosity are basically unaffected by the approximation. The situation is more complex in dendrimers, because of the larger hydrodynamic interaction. Therefore, the full numerical diagonalization is by far the simplest and the fastest procedure $[19,31]$. In this connection, we recall that the main source of error of the present treatment is due to the preaveraging approximation, in view of the large bead density [52].

The intrinsic viscosity is obtained from equations (2.21) and (2.22). As done 


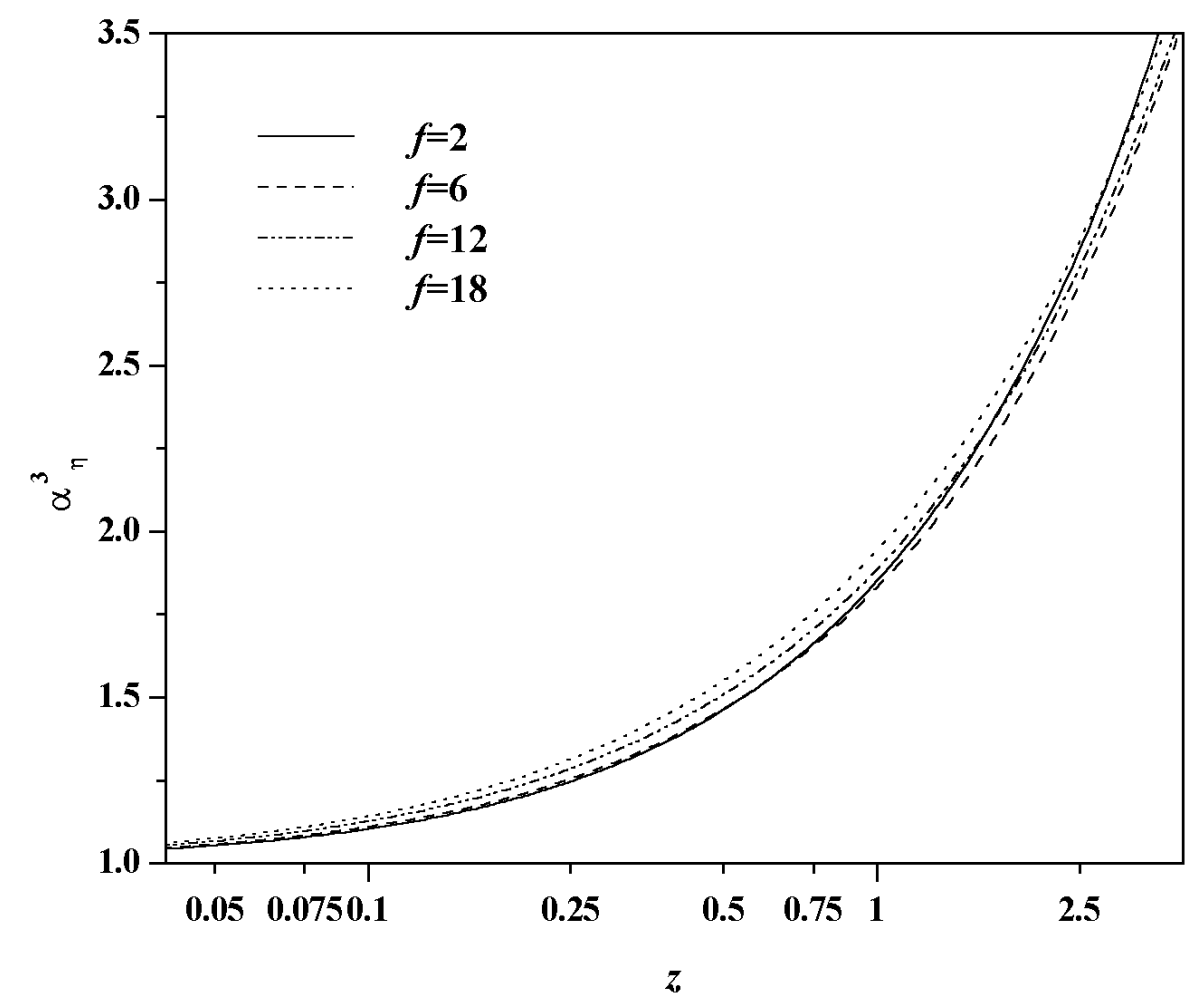

Figure 9. Universal plots of $\alpha_{\eta}^{3}$ vs. the reduced variable $z=\tau B \sqrt{N}$ for linear $(f=2)$ and $\operatorname{star}(f=6,12,18)$ polymers in a good solvent.

before for the radius of gyration, we define the expansion factor of the intrinsic viscosity with respect to the random-walk conformation:

$$
\alpha_{\eta}^{3}=[\eta] /[\eta]^{\mathrm{RW}}
$$

the third power arising from the dimensions of intrinsic viscosity (volume/mass). In stars, for a given choice of the reduced friction coefficient per bead $\zeta_{r}, \alpha_{\eta}^{3}$ is a universal function of $z$ [26]. The GSC results reported in figure 9, are well fitted by the analytical expressions

$$
\begin{array}{ll}
\alpha_{\eta}^{3}=\left[1+2.804 z+4.095 z^{2}\right]^{3 / 10} & (f=2, \text { linear }), \\
\alpha_{\eta}^{3}=\left[1+3.364 z+3.216 z^{2}\right]^{3 / 10} & (f=6), \\
\alpha_{\eta}^{3}=\left[1+4.156 z+3.171 z^{2}\right]^{3 / 10} & (f=12), \\
\alpha_{\eta}^{3}=\left[1+4.822 z+3.376 z^{2}\right]^{3 / 10} & (f=18) .
\end{array}
$$

The exponent outside the square brackets was chosen so as to asymptotically give Zimm's result $[\eta] \propto N^{0.8}[9,30]$, whereas the perturbative results cannot be reproduced because of our use of the Gaussian approximation in equation (2.17).

We also introduce the topological ratio of the intrinsic viscosity for star polymers:

$$
g_{\eta}=[\eta]_{\text {star }} /[\eta]_{\text {lin }}=g_{\eta}^{\mathrm{RW}} \cdot \alpha_{\eta, \mathrm{star}}^{3} / \alpha_{\eta, \operatorname{lin}}^{3} \cdot
$$




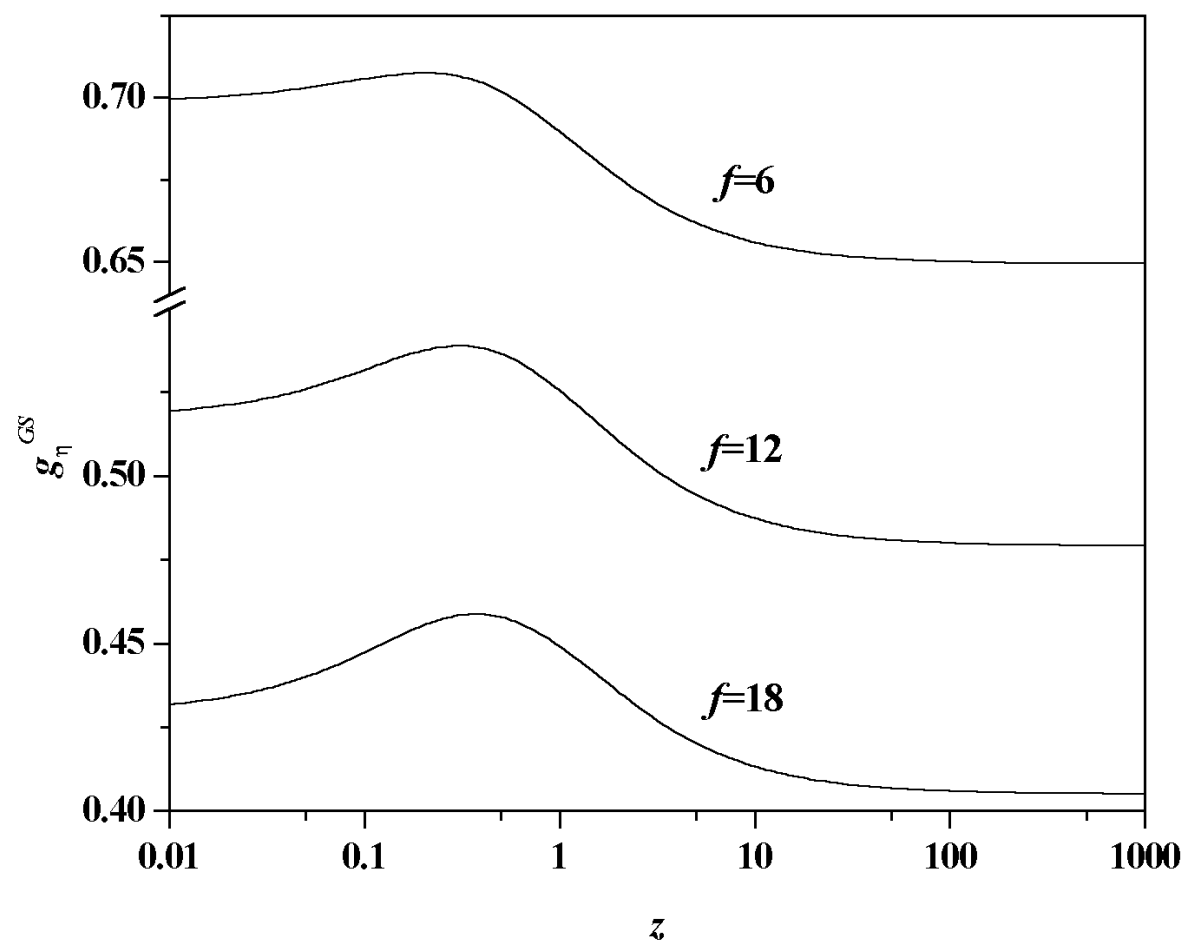

Figure 10. The topological ratio of the intrinsic viscosity $g_{\eta}^{\text {GS }}$ plotted as a function of the reduced variable $z=\tau B \sqrt{N}$ for star polymers in a good solvent.

Here, $g_{\eta}^{\mathrm{RW}}$ is the RW result, first obtained by Zimm and Kilb [50]: its numerical values can be found in [33]. The $g_{\eta}^{\mathrm{GS}}$ ratio is reported in figure 10 as a function of $z$. The initial slight increase of $g_{\eta}^{\text {GS }}$ qualitatively agrees with the perturbative results, while the asymptotic decrease by about $6 \%$ to a constant value for all stars, although obtained by extrapolation, is consistent with experimental results [5].

In dendrimers, $\alpha_{\eta}$ is predicted to show a cross-over to a constant plateau value with increasing molecular expansion, just as found for $\alpha_{\mathrm{S}}$. A universal curve was obtained using as universal variable $\tau B N^{\xi}$, with $\xi=0.735$, and the full curve, shown in figure 11, was again fitted by a saturation Hill curve of the same form as in equation (4.7). Note that the $\xi$ exponent is significantly lower than the corresponding $\varphi$ exponent required for $\alpha_{S}$. While we have no simple explanation for this difference, we point out that relevant factors are the finite size of the dendra and the complex hydrodynamic behaviour within these overcrowded molecules. As concerns the effect of the limited dendron length, we point out that we extracted apparent Flory exponents from the relationship $2 \nu_{\text {app }}=\partial\left\langle R_{\mathrm{S}}^{2}\right\rangle / \partial n_{\text {beads }} . \nu_{\text {app }}$ steadily decreases from 0.43 for $g=1$ to 0.23 for $g=6$ at intermediate solvent quality [19], in reasonable agreement with simulations [53,54]. No simple power law can thus be obtained for dendrimers of a realistic generation, as anticipated. If we were now to use the scaling relationship $[\eta] \propto R^{3} / n_{\text {beads }}$ through some appropriate radius $R$ proportional to the radius of gyration [53], the above exponents would yield $[\eta] \propto n_{\text {beads }}^{3 \nu_{\text {app }} \text {, }}$, which increases as $n_{\text {beads }}^{0.29}$ at low $g$, but decreases as $n_{\text {beads }}^{-0.31}$ at large $g$. Therefore, $[\eta]$ 


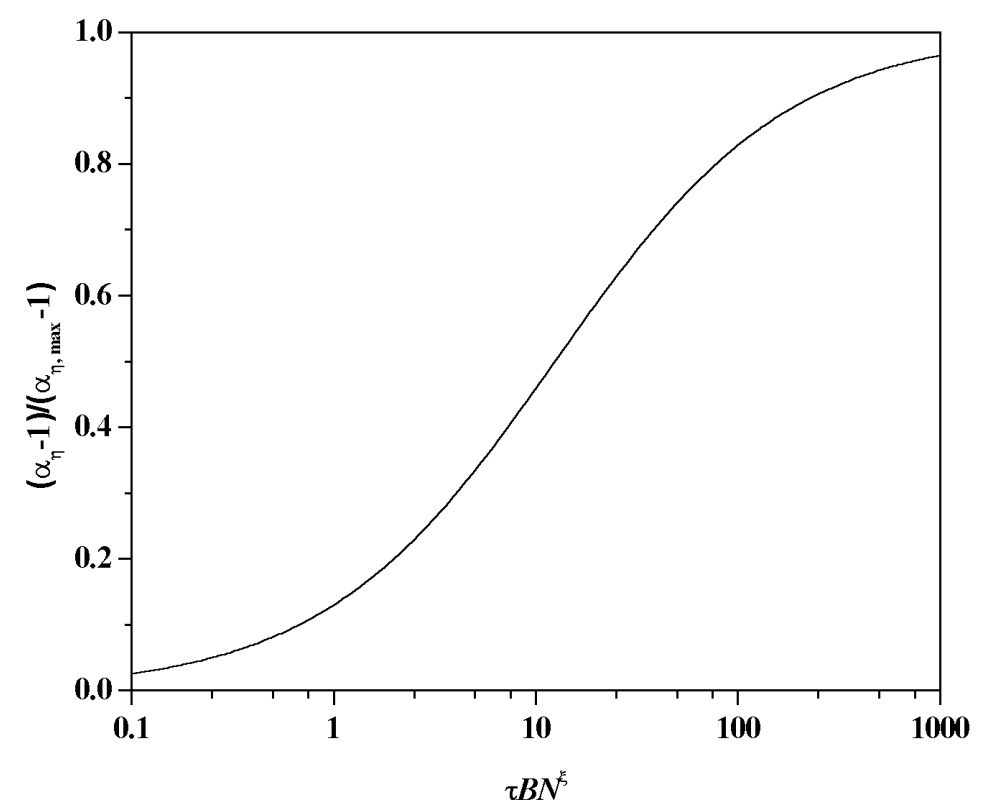

Figure 11. Universal plot of the expansion factor of the intrinsic viscosity for the dendrimers of figure $1 \mathrm{~b}$ in a good solvent plotted as $\left(\alpha_{\eta}-1\right) /\left(\alpha_{\eta, \max }-1\right)$ vs. the reduced variable $\tau B N^{\xi}$. Here $\xi=0.735$, and $\alpha_{\eta \text {, max }}$ is the asymptotic value at maximum expansion of the molecule with fixed bond lengths [19], while the $H$ and $x_{1 / 2}$ parameters of equation (4.7) have the best-fit values $H=0.787$ and $x_{1 / 2}=11.19$.

should pass through a maximum, as indeed found experimentally in some cases [2]. However, the full calculation does not support this result, and indicates that $[\eta]$ monotonously increases with $g$. If the latter result is affected by the preaveraging approximation, we point out that because of the limited dendron span, simple and general scaling relationships are ruled out.

Let us now turn to dynamical-mechanical experiments. Figure 12 shows results for the complex modulus of stars in a good solvent [55] with an increasing number of arms and a fixed arm length. At low frequency, we recover the usual power-laws $G^{\prime} \propto \omega^{2}$ and $G^{\prime \prime}-\omega \eta_{0} \propto \omega$. The intramolecular dynamics, where $G^{\prime} \propto G^{\prime \prime}-\omega \eta_{0} \propto$ $\omega^{1 / \beta}$ [see equation (2.25)], is observed for $\tau_{1}^{-1}<\omega<\tau_{N}^{-1}$, the upper frequency roughly corresponding to the crossing of the curves of $G^{\prime}$ and $G^{\prime \prime}$. The length of this interval is about the same for stars with the same arm length: on the other hand, for stars with the same molar mass this interval progressively shrinks with an increasing number of arms because of their corresponding shortening $[6,55,56]$. The $\beta$ exponent is very close to the theoretical good-solvent value of $5 / 9$, but the main feature differentiating linear and star polymers consists in the shoulder at $\omega \tau_{1} \cong 1$ for $G^{\prime \prime}$ in the stars, in particular for $f=12$. This shoulder, that may become a shallow maximum at larger $f$, is due to the $f-1$ multiplicity of the first mode that strongly enhances its contribution compared to that due to the non-degenerate second mode [55].

The oscillatory flow birefringence predicted for linear and star polymers is shown 


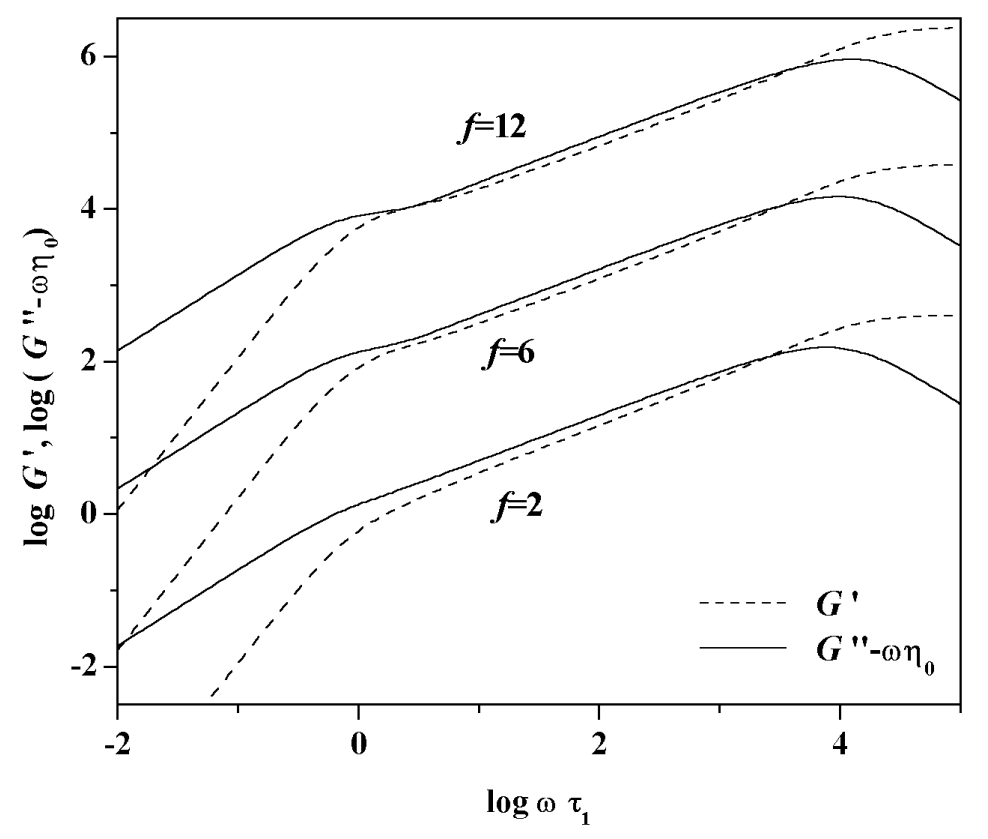

Figure 12. The real and imaginary parts of the complex modulus $G^{\prime}$ and $G^{\prime \prime}-$ $\omega \eta_{0}$ (the storage and loss modulus) in $c R T / M$ units plotted as a function of the applied frequency $\omega$ for linear $(f=2)$ and star $(f=6,12)$ polymers in a good solvent. On the abscissa, the frequency was scaled through the longest intramolecular relaxation time $\tau_{1}$. For the sake of clarity, the curves for the 6arm star were rigidly shifted upwards by 1.5 , and those for the 12-arm star by 3 .

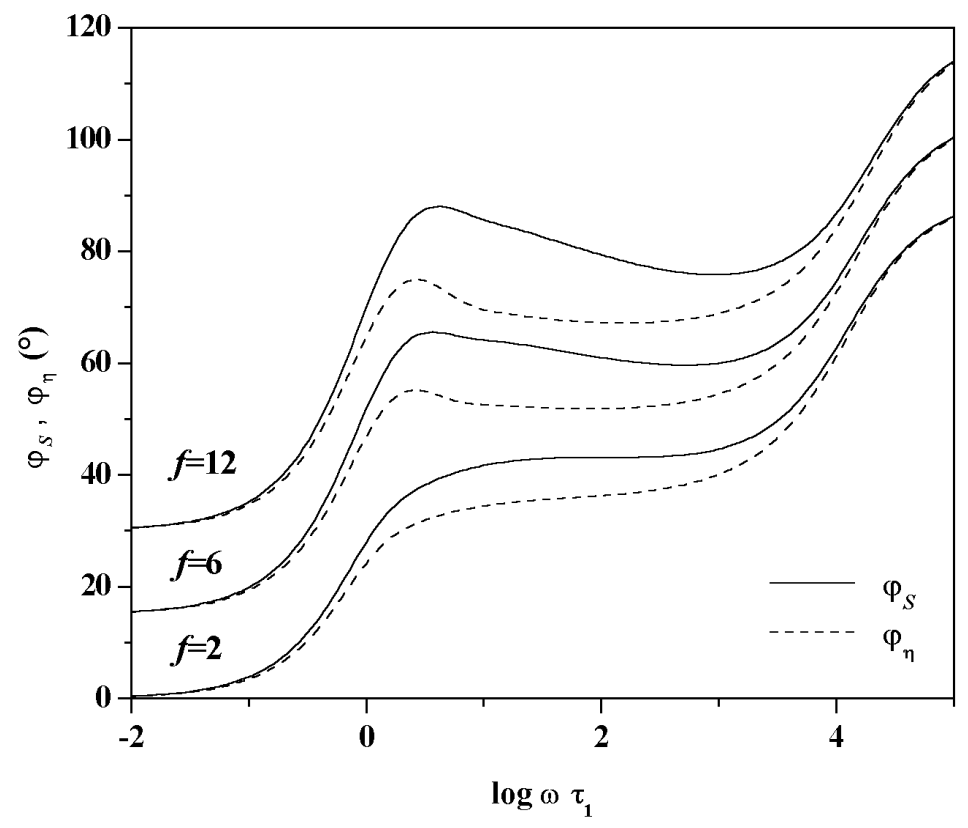

Figure 13. The phase angles $\varphi_{\mathrm{S}}$ and $\varphi_{\eta}$ for the oscillatory flow birefringence and the dynamic viscosity as a function of the applied frequency as in figure 12 (see also the text). For the sake of clarity, the curves for the 6 -arm star were rigidly shifted upwards by $15^{\circ}$, and those for the 12 -arm star by $30^{\circ}$. 


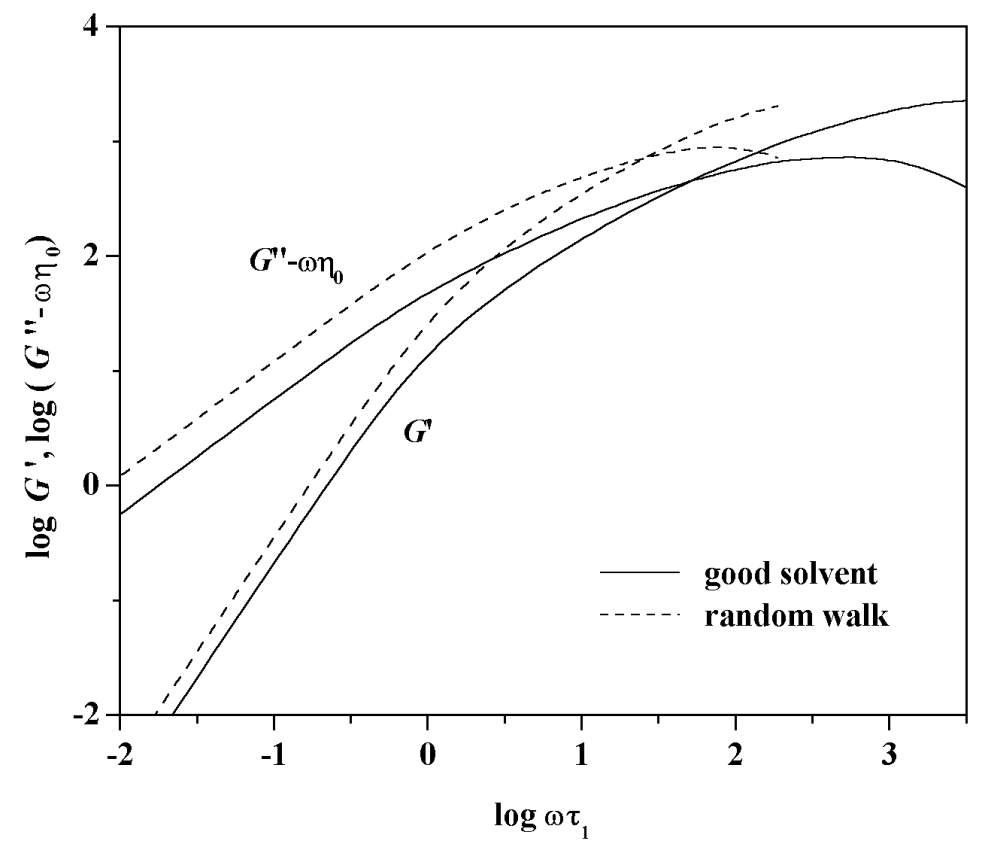

Figure 14. The real and imaginary parts of the complex modulus $G^{\prime}$ and $G^{\prime \prime}-\omega \eta_{0}$ (the storage and loss modulus) in $c R T / M$ units plotted as a function of the applied frequency $\omega$ for the 6th generation dendrimer shown in figure $1 \mathrm{~b}$. On the abscissa, the frequency was scaled through the longest intramolecular relaxation time $\tau_{1}$.

in figure 13 through the frequency dependence of the phase angle $\varphi_{\mathrm{S}}$ in comparison with the analogous quantity $\varphi_{\eta}$ of the dynamic viscosity. These phase angles are very sensitive to solvent conditions, and give rise to a well-defined maximum in stars matching the shoulder of $G^{\prime \prime}-\omega \eta_{0}$. A noteworthy feature consists in the clear difference between $\varphi_{\mathrm{S}}$ and $\varphi_{\eta}$. In fact, the two experiments provide different information, since dynamic viscosity probes the orientation of intramolecular forces with the applied deformation, whereas optical flow birefringence measures the orientation of bonds. In the bead-and-spring model with a RW conformation the forces acting on a bead are only transmitted by the adjacent bonds, and accordingly, the two experiments are entirely equivalent, with $\varphi_{\mathrm{S}} \equiv \varphi_{\eta}$. Conversely, in a good solvent the intramolecular forces depend on all the bonds, in principle, because of the long-range correlations, and therefore we expect $\varphi_{\mathrm{S}} \neq \varphi_{\eta}$.

The complex modulus calculated for dendrimers is shown in figure 14. Here, it is difficult to unambiguously detect the region of intramolecular dynamics because of the limited dendron length and the smooth crossover with the low frequency response where $G^{\prime} \propto \omega^{2}$ and $G^{\prime \prime}-\omega \eta_{0} \propto \omega$. A linear portion in the doubly logarithmic plot is only seen in high-generation dendrimers at large expansion, in particular when the molecule comprises two bonds between adjacent branch points (not shown here). In the latter case, the slope in the log-log plot is marginally smaller than in star polymers [31], but it cannot be related to any exponent of the relaxation times because in dendrimers these do not follow a power-law dependence on the mode index. 


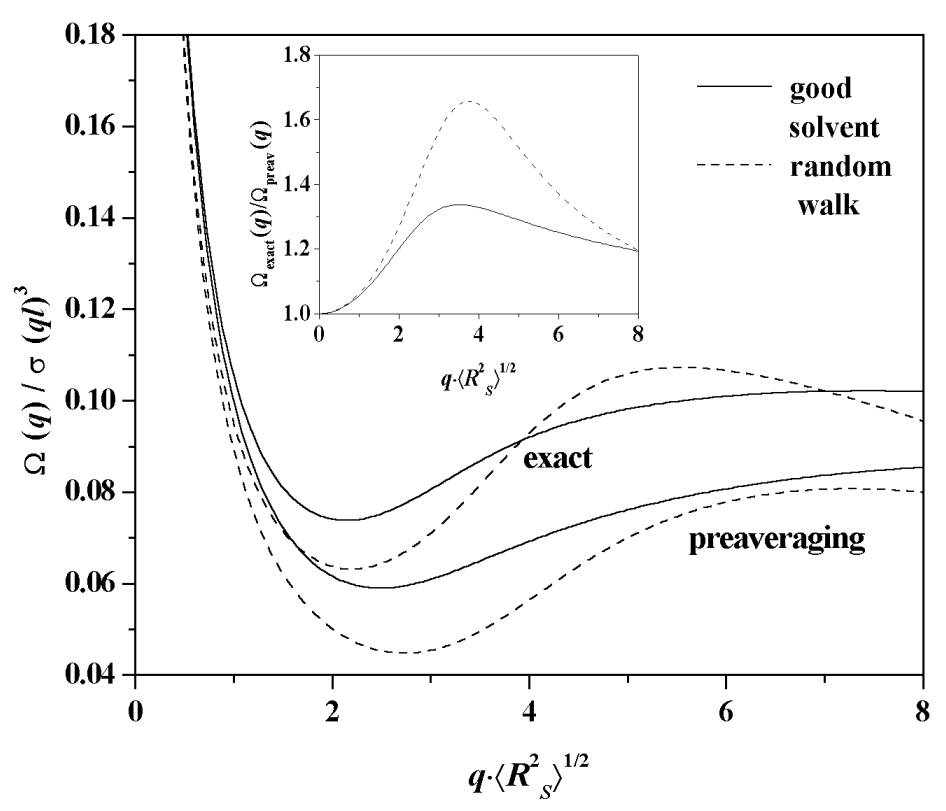

Figure 15. The first cumulant for the 6 th generation dendrimer shown in figure $1 \mathrm{~b}$ plotted as $\Omega / \sigma \cdot(q l)^{3}$ as a function of the modulus of the scattering vector $q$ normalized by the root-mean-square radius of gyration. The time unit is $\sigma^{-1}$ and $l$ is the bond length. The inset shows the error of the first cumulant $\Omega_{\text {exact }} / \Omega_{\text {preav }}$, "exact" and "preav" referring to the calculations without preaveraging and with preaveraging the hydrodynamic interaction, within the GSC approach.

Finally, we discuss the first cumulant of the dynamic structure factor because it enables us to gauge the error entailed by the preaveraging approximation. Since this error becomes larger with increasing bead density [52], we report here our results for dendrimers only [31]. The first cumulant $\Omega(q)$ is reported in figure 15 as a function of $q$. Both at very small and at very large $q$, we have $\Omega(q) \propto q^{2}$ due to the diffusive motion either of the whole molecule (low $q$ ) or of the single bead (high $q$ ) before it experiences the connectivity constraints [30,34]. In the intermediate $q$ range the dependence switches to $\Omega(q) \propto q^{3}$. Note that these exponents are insensitive to good-solvent expansion, that merely modifies the prefactors. The characteristic feature of branched polymers consists of a shallow minimum in the plot of the normalized cumulant $\Omega(q) / q^{3}$ at about $q \cdot\left\langle R_{\mathrm{S}}^{2}\right\rangle^{1 / 2} \cong 2 \div 3$, as predicted and observed in stars $[15,57]$, matching the corresponding maximum in the Kratky plot of the static structure factor $S(q)$ [58]. Such minimum, that is absent in linear chains, is more pronounced in dendrimers due to the larger local density, and therefore it becomes deeper with increasing generation, and shallower with good-solvent expansion. The region of the minimum is also the $q$ range where the preaveraging approximation is mostly in error, as shown in the inset. Interestingly, good-solvent expansion makes the error significantly smaller, in keeping with the notion that the molecules display an intermediate draining regime, in particular with more bonds between adjacent branch points because of the lower local density. Finally, we note that the preaveraging error amounts to underestimate $\Omega(q)$, i.e. to predict a slower 
intramolecular relaxation. In turn, this produces a larger $[\eta]$ via longer relaxation times, which is a well-known result [52].

\section{Star polymers in a poor solvent and amphiphilic block-co- polymer stars}

The poor-solvent conditions are realized at $T<\Theta$, when the two-body interactions among the beads of the same or of different molecules become attractive. As a result, there is competition between intramolecular collapse to a globule and intermolecular aggregation with eventual precipitation [21]. While the kinetics of these processes dictates what the exact fate of each molecule is, extremely dilute solutions and a viscous solvent strongly favour the monomolecular process. At a low undercooling below $\Theta$, collapse to the globular state is resisted by configurational entropy, while in the globule the three-body repulsions dominate, dictating also its density. In water-soluble polymers the poor-solvent region is usually found at $T>\Theta$ when the hydrogen bonds with solvent are disrupted, but the present argument does not change, apart from some trivial redefinition of the $b_{2}$ parameter.

In the globular state, we expect the mean-square radius of gyration to follow equation (1.2) with a Flory exponent $\nu=1 / 3$, so that the density within the molecule is independent of molar mass $[20,21]$. The parameter of the three-body interactions $b_{3}$ determines both the transition temperature and the transition order to a globule. For a small $b_{3}$, corresponding to thin and long bonds, we have a first-order transition, and two sharply different molecular conformations coexist at the transition temperature, one being close to unperturbed, and the other one being close to a compact globule. Conversely, a large $b_{3}$ yields a smooth, second-order transition. An extensive analysis of these issues is to be found in [21] and [59], where we discuss the thermodynamic meaning of these transition orders and their peculiarities related with the finite size of the molecules and with molecular connectivity.

The behaviour of amphiphilic block-copolymer stars in selective solvents is definitely of greater interest [60]. For the sake of argument, we assume the solvent to be water, and the star arms to be made by two blocks of equal length comprising hydrophobic beads (H-beads) and polar beads (P-beads). Therefore, the H-beads are in a poor solvent and tend to cluster together, while the P-beads are in a good solvent, well solvated by the water molecules. We consider copolymer stars with both topological connections: INNER-H stars, where the $\mathrm{H}$ block is connected to the core and the P-beads are on the outside, and OUTER-H stars, with the opposite topology. Within the present approach, the nature of the beads and their polarity or hydrophobicity are simply characterized by the parameter $B_{i}= \pm \sigma$, a positive sign indicating a P-beads, and a negative one indicating an $\mathrm{H}$-bead. The pairwise interactions are expressed as $B_{i j}=\left(B_{i}+B_{j}\right) / 2$, which replaces the $b_{2}$ coefficient within the double sum of $\mathcal{A}_{2}$ in equation (2.8) [60]. Therefore, $\sigma$ measures the amphiphilicity of the molecule. The two-body interactions between a $\mathrm{P}-$ and an H-bead vanish, while all beads are still subject to three-body repulsions, whose $b_{3}$ parameter is taken as independent from the bead triplet. In order to test 

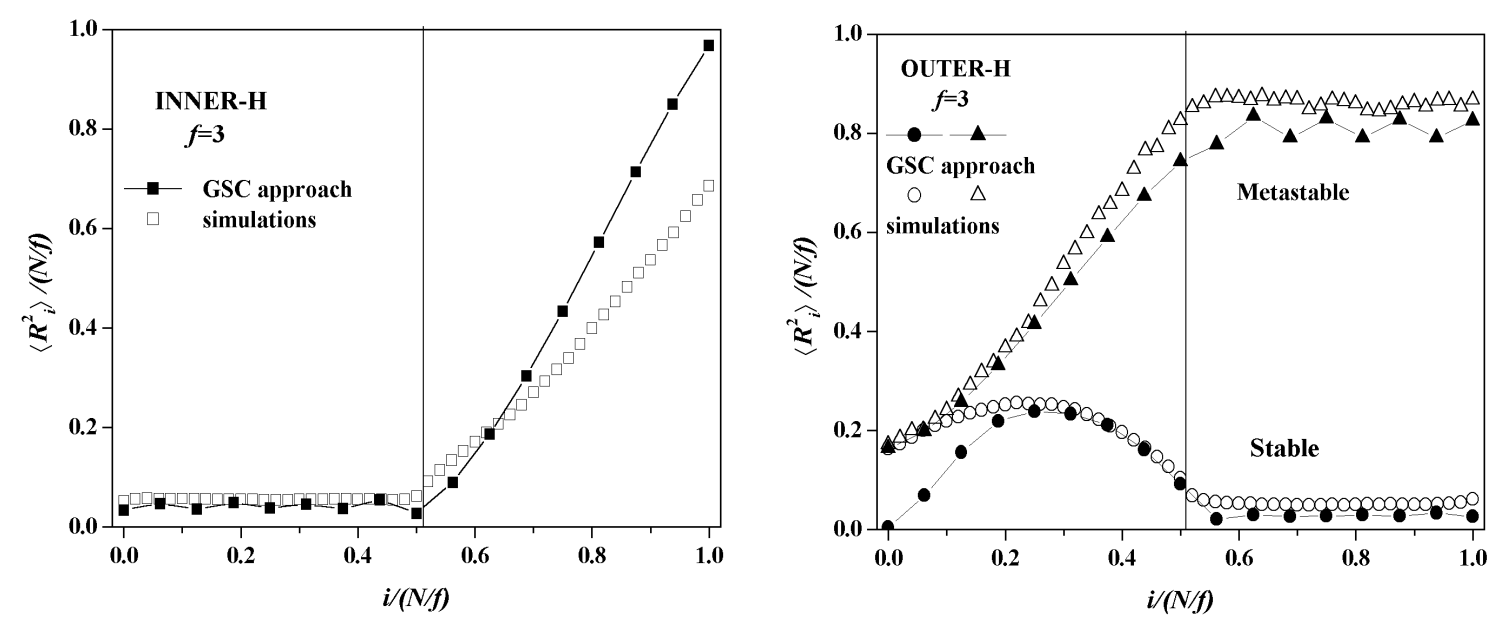

Figure 16. The mean-square distances of the beads from the molecular center of mass $\left\langle R_{i}^{2}\right\rangle, i=0,1,2, \ldots, N / f$ for INNER-H (left) and OUTER-H (right) 3 -arm stars as a function of their position along the arm ( 0 is the branch point and $N / f$ the free end). Both the vertical and the horizontal axis were normalized by the number of beads per arm to quantitatively compare the GSC and the simulation results $(N / f=16$ and 51 , respectively). The vertical line separates the P-beads from the H-beads (the latter ones being given by $i /(N / f)<0.5$ in the INNER-H star and by $i /(N / f)>0.5$ in the OUTER-H star $)$.

the GSC approach, off-lattice Monte Carlo simulations were carried out [60] using a hamiltonian that includes a harmonic potential for the connected beads, and a Lennard-Jones potential for the non-bonded interactions. The energy parameter of the latter potential was introduced as in the GSC approach, and the simulations swept the range of composition from $20 \%$ to $80 \%$ of H-beads, although the overall picture was qualitatively unchanged.

With increasing degree of amphiphilicity $\sigma$, both INNER-H and OUTER-H stars form a compact globule comprising all the H-beads wherefrom the P-beads are expelled, surrounding it in a sort of monomolecular micelle. Thus, intermolecular aggregation is basically forbidden because of the mutually repulsive outer beads, which provide a sort of colloidal stabilization. In the INNER-H star, the overall molecular size is larger because of the free ends, while in the OUTER-H star the P-blocks, connected also to the core bead, form long loops at the globular surface. However, in the OUTER-H star the picture is richer, because a non-trivial metastable state is also possible. In such a state, the inner P-beads keep apart the arms, and the H-blocks form small sub-globules at the end of each arm. These long-lived droplets constitute a robust local minimum, their coalescence to the single globule of the stable state being kinetically difficult. Only at very large amphiphilicity a spinodal regime sets in, rendering such conformation unstable. Obviously enough, the metastable conformation does not provide any colloidal stabilization against intermolecular aggregation and phase separation [60].

The agreement of the GSC results with the Monte Carlo simulation is quite good even from the quantitative viewpoint, as shown for instance by the mean-square dis- 
tances of the beads from the molecular center of mass $\left\langle R_{i}^{2}\right\rangle, i=0,1,2, \ldots, N / f$ for both INNER-H and OUTER-H stars, reported in figure 16. The results quantitatively agree in general, apart from minor effects, the only significant difference being the GSC prediction that the core P-bead should be trapped within the compact hydrophobic globule in the stable state of the OUTER-H star. Such trapping is not borne out by the simulations, and therefore it is an artifact of the GSC approach, that accounts only for the average molecular conformation through quadratic averages. Among the other calculated quantities, the density profiles $\rho(r)$ of the $\mathrm{H}$ - or P-beads and of the undifferentiated beads as a function of the distance $r$ from the molecular center of mass, display the main shortcoming of the Gaussian approximation. In fact, the agreement between the GSC and the Monte Carlo result for $\rho(r)$ is reasonably good, apart from the density profile of the P-beads close to the center of mass. According to the simulations, this profile vanishes at a finite $r>0$ since these beads are expelled from the globular core. Conversely, this may not happen in the GSC approach, that yields a vanishing radial density profile only for $r \rightarrow 0$ (or $r \rightarrow \infty$ ): the Gaussian distribution function cannot produce any flat density profile, as it is well known. We stress, however, that apart from this shortcoming, the GSC approach produces quantitatively correct results in most cases with the simplest mathematical formulation.

\section{Concluding remarks}

In this paper, we review some configurational and dynamical properties of regular branched polymers within the Gaussian Self-Consistent approach. The method allows us to investigate both equilibrium and dynamics within a unified, consistent method and is has many advantages: i) it correctly accounts for all the configurational degrees of freedom, which is of major importance; ii) it explicitly accounts for molecular connectivity; iii) it provides detailed information about the intramolecular conformation in terms of quadratic averages or through the average scalar products among the bond vectors; iv) it permits studying molecules of any topology; v) it allows specifying the detailed sequence of any number of unlike beads in random or block copolymers; vi) it is naturally consistent with the linear form of the stochastic Langevin equation in dynamics. Some of these features are clearly interdependent, but they show the rich potentiality of the method.

The main approximation of the approach consists in adopting the Gaussian approximation for the distribution function of the bond vectors connecting adjacent beads and of the distances among the beads. A related problem in dynamics consists in the preaveraging approximation for the hydrodynamic interaction and in expressing the reciprocal averages through the Gaussian distribution, which is particularly in error for topologically close beads. Improving our approach beyond the Gaussian approximation is not trivial. The general form of the correct distribution function is known for linear chains [14], but it is not clear whether it applies also in the presence of branch points. Also, it does not produce a linear elastic force, so that the linear Langevin equation cannot be used any more for the intramolecular dy- 
namics, rendering the consistent treatment of equilibrium and dynamics a daunting task. Incidentally, we note that in linear chains the renormalization group results for dynamic properties compare less favourably with experiments than the GSC predictions [61]. Computer simulations do not suffer from the above limitations and indeed provided much insight in the behaviour of branched polymers. In this case, a different problem arises regarding the ergodicity of the simulations, because of the limitations to the accessible conformations and/or to the local or global motions allowed by the steric interactions. In particular, an efficient sampling of the phase space may become a serious problem in high-generation dendrimers.

Other effects in a dilute polymer solution can also be investigated within the GSC approach, such as the shear deformation and the solution viscosity at large shear rate $\dot{\gamma}$ [62]. Actually, since in the bead-and-spring model each "bond" can be stretched without limit with increasing $\dot{\gamma}$, a constraint must be applied to the average meansquare spring lengths. The latter constraint amounts to a stiffening of the springs, as expected in real molecules when the local conformation is fully elongated. With this additional feature, we could describe the elongation and orientation of linear and star polymers with increasing $\dot{\gamma}$ in terms of a prolate ellipsoid forming the molecular envelope [62]. We also calculated the intrinsic viscosity, predicting a nonNewtonian behaviour with first a slight shear thinning, then thickening taking place at $\dot{\gamma} \approx \tau_{\dot{\gamma}}^{-1}$, where $\tau_{\dot{\gamma}}$ is a characteristic time of the molecule, and eventually a much larger thinning described by the power law $[\eta] \propto \dot{\gamma}^{-2 / 3}$. Interestingly, the model predicts that good-solvent effects and hydrodynamic interaction become irrelevant at a large shear rate because of the molecular elongation, in keeping with simple expectation.

From the experimental viewpoint, we mention the issue of the unperturbed state of dendrimers, that was hardly investigated, to the best of our knowledge. Experiments on such systems will provide an even more stringent test of the theoretical prediction about the unperturbed state than provided by stars. The rheological behaviour of dendrimers at a large shear rate is another aspect still needing extensive studies, that may reveal potentially important features of these fascinating molecules.

\section{Acknowledgements}

We gratefully thank Prof. G.Allegra, who introduced us to polymer physics, for many useful discussions and encouragement. We also thank Dr. Guido Raos, Dr. Giuseppina Raffaini and Dr. Roberto La Ferla for stimulating discussions and fruitful collaboration. We are grateful to Dr. E.G.Timoshenko and Dr. Kuznetsov, of the University College Dublin (Ireland), for illuminating discussions. This work was financially supported by MURST (Ministry of University and of Scientific and Technological Research, Italy) and by CNR (Consiglio Nazionale delle Ricerche, Italy) Project "Materiali Speciali per Tecnologie Avanzate II". 


\section{References}

1. Grest G.S., Fetters L.J., Huang J.S., Richter D. Star polymers: experiment, theory and simulation. // Adv. Chem. Phys., 1996, vol. 94, p. 67-163.

2. Tomalia D.A., Naylor A.M., Goddard W.A. III Starbust dendrimers: molecular-level control of size, shape, surface chemistry, topology, and flexibility from atoms to macroscopic matter. // Angew. Chem. Int. Ed. Engl., 1990, vol. 29, p. 138-175.

3. Branched polymers I and II, edited by Roovers J. // Adv. Polym. Sci., 1999, vol. 142 and 143.

4. Trollsås M., Atthorf B., Würsch A., Hedrick J.L., Pople J.A., Gast A.P. Constitutional isomers of dendrimer-like star polymers: design, synthesis, and conformational and structural properties. // Macromolecules, 2000, vol. 33, No. 17, p. 6423-6438.

5. Douglas J.F., Roovers J., Freed K.F. Characterization of branching architecture through "universal" ratios of polymer solution properties. // Macromolecules, 1990, vol. 23, No. 18 , p. $4168-4180$.

6. Freire J.J. Conformational properties of branched polymers: theory and simulations. // Adv. Polym. Sci., 1999, vol. 143, p. 35-112.

7. Bosman A.W., Janssen H.M., Meijer E.W. About dendrimers: structure, physical properties, and applications. // Chem. Rev., 1999, vol. 99, No. 7, p. 1665-1688.

8. de Gennes P.-G., Hervet H. Statistics of "starburst" dendrimers. // J. Physique Lettres, 1983, vol. 44, No. 9, p. L-351-L-360.

9. Allegra G., Ganazzoli F. Chain configuration and dynamics in the Gaussian approximation. // Adv. Chem. Phys., 1989, vol. 75, p. 265-348.

10. Timoshenko E.G., Dawson K.A. Equilibrium properties of polymers from the Langevin equation: Gaussian self-consistent approach. // Phys. Rev., 1995, vol. E 51, No. 1, p. 492-498; Kuznetsov Yu.A., Timoshenko E.G., Dawson K.A. Kinetics at the collapse transition of homopolymers and random copolymers. // J. Chem. Phys., 1995, vol. 103, No. 11, p. 4807-4818; Kuznetsov Yu.A., Timoshenko E.G. Self-consistent treatment of copolymers with arbitrary sequences. // Il Nuovo Cimento, 1998, vol. 20D, No. 12bis, p. 2265-2288; Kuznetsov Yu.A., Timoshenko E.G. On the conformational structure of a stiff homopolymer. // J. Chem. Phys., 1999, vol. 111, No. 8, p. 3744-3752.

11. La Ferla R. Conformations and dynamics of dendrimers and cascade molecules. // J. Chem. Phys., 1997, vol. 106, No. 2, p. 688-700.

12. Flory P.J. Principles of Polymer Chemistry. Ithaca (NY), Cornell University Press, 1953.

13. Yamakawa H. Modern Theory of Polymer Solutions. New York, Harper \& Row, 1971.

14. de Gennes P.-G. Scaling Concepts in Polymer Physics. Ithaca (NY), Cornell University Press, 1979.

15. Burchard W. Static and dynamic light scattering from branched polymers and biopolymers. // Adv. Pol. Sci., 1983, vol. 48, p. 1-124.

16. Batoulis J., Kremer K. Thermodynamic properties of star polymers: good solvent. // Macromolecules, 1989, vol. 22, No. 11, p. 4277-4285.

17. Zifferer G. Monte Carlo simulation studies of the size and shape of linear and starbranched polymers embedded in the tetrahedral lattice. // Macromol. Theory Simul., 1999, vol. 8, No. 5, p. 433-462.

18. Zimm B.H., Stockmayer W.H. The dimensions of chain molecules containing branches and rings. // J. Chem. Phys., 1949, vol. 17, No. 12, p. 1301-1314. 
19. Ganazzoli F., La Ferla R., Terragni G. Conformational properties and intrinsic viscosity of dendrimers under excluded volume conditions. // Macromolecules, 2000, vol. 33, No. 17, p. 6611-6620.

20. Grosberg A.Yu., Khokhlov A.R. Statistical Physics of Macromolecules. New York, AIP Press, 1994.

21. Ganazzoli F., Raos G., Allegra G. Polymer association in poor solvents: from monomolecular micelles to clusters of chains and phase separation. // Macromol. Theory Simul, 1999, vol. 8, No. 1, p. 65-84.

22. Ganazzoli F. Collapse of random copolymers. // J. Chem. Phys., 1998, vol. 108, No. 23, p. 9924-9932; Globular state of random copolymers with arbitrary amphiphilicity. // J. Chem. Phys., 2000, vol. 112, No. 3, p. 1547-1553.

23. Allegra G., Ganazzoli F. The conformation of linear and star polymers in solution. // Prog. Polym. Sci., 1991, vol. 16, p. 463-508.

24. Ganazzoli F. Two- and three-body interactions in the $\Theta$ state: linear and star polymers. // Macromolecules, 1992, vol. 25, No. 26, p. 7357-7364.

25. Ganazzoli F., La Ferla R. The unperturbed state of dendrimers. // J. Chem. Phys., 2000, vol 113, No. 20, p. 9288-9293.

26. Allegra G., Colombo E., Ganazzoli F. Linear and regular star polymer in a good solvent. // Macromolecules, 1993, vol. 26, No. 2, p. 330-338.

27. Zimm B.H. Dynamics of polymer molecules in dilute solution: viscoelasticity, flow birefringence and dielectric loss. // J. Chem. Phys., 1956, vol. 24, No. 2, p. 269-278.

28. Guenza M., Perico A. A reduced description of the local dynamics of star polymers. // Macromolecules, 1992, vol. 25, No. 22, p. 5942-5949.

29. Bixon M. Dynamics of polymer molecules in dilute solution. // J. Chem. Phys., 1973, vol. 58, No. 4, p. 1459-1466; Zwanzig R. Theoretical basis for the Rouse-Zimm model in polymer solution dynamics. // J. Chem. Phys., 1974, vol.60, No. 7, 2717-2720; Bixon M., Zwanzig R. Optimized Rouse-Zimm theory for stiff polymers. // J. Chem. Phys., 1978, vol. 68, No. 4, 1896-1902.

30. Doi M., Edwards S.F. The Theory of Polymer Dynamics. Oxford, Oxford Scientific Publications, 1986.

31. Ganazzoli F., La Ferla R., Raffaini G. Intramolecular dynamics of dendrimers under excluded volume conditions. // Macromolecules, 2001, vol. 34, No. 12, p. 4222-4228.

32. Perico A. Segmental relaxation in macromolecules. // Acc. Chem. Res., 1989, vol. 22, No. 9, p. 336-342.

33. Ganazzoli F., Allegra G., Colombo E., De Vitis M. Dynamics of regular star polymers: the intrinsic viscosity. // Macromolecules, 1995, vol. 28, No. 4, p. 1076-1084.

34. Akcasu Z., Gurol H. Quasielastic scattering by dilute polymer solutions. // J. Pol. Sci., Pol. Phys. Ed., 1976, vol. 14, p. 1-10.

35. Bauer B.J., Hadjichristidis N., Fetters L.J., Roovers J.E.L. Star-branched polymers. 5. The $\Theta$ temperature depression for 8- and 12-arm polyisoprenes in dioxane. // J. Am. Chem. Soc., 1980, vol. 102, No. 7, p. 2410-2413.

36. Cherayil B.J., Douglas J.F., Freed K. F. Effect of residual interactions on polymer properties near the theta point. // J. Chem. Phys., 1985, vol. 83, No. 10, p. 52935310; Effect of residual interactions on polymer properties near the theta point. II. Higher moments and comparison with Monte Carlo simulations. // J. Chem. Phys., 1987, vol. 87, No. 5, p. 3089-3098.

37. Tanaka F. Osmotic pressure of ring-polymer solutions. // J. Chem. Phys., 1987, vol. 87, 
No. 7, p. 4201-4206; Iwata K. $\Theta$ temperature of ring polymers: another evidence of topological interaction. // Macromolecules, 1989, vol. 22, No. 9, p. 3702-3706; Roovers J., Toporowski P.M. Synthesis of high molecular weight ring polystyrenes. // Macromolecules, 1983, vol. 16, No. 6, p. 843-849.

38. Candau P., Rempp P. Benoit H. A new theoretical approach to the problem of solution behaviour of branched polymers. // Macromolecules, 1972, vol. 5, No. 5, p. 627-635.

39. Roovers J., Hadjichristidis N., Fetters L.J. Analysis and dilute solution properties of 12- and 18-arm star polystyrenes. // Macromolecules, 1983, vol. 16, No. 2, p. 214220; Huber K., Burchard W., Fetters L.J. Dynamic light scattering from regular starbranched molecules. // Macromolecules, 1984, vol. 17, No. 4, p. 541-548.

40. Ganazzoli F., Allegra G., Raos G. The conformation of linear and star polymers in the ideal state. // Gazz. Chim. Ital., 1996, vol. 126, p. 779-782.

41. Horton J.C., Squires G.L., Boothroyd A.T., Fetters L.J., Rennie A.R., Glinka C.J., Robinson R.A. Small-angle neutron scattering from star-branched polymers in the molten state. // Macromolecules, 1989, vol. 22, No. 2, p. 681-686; Boothroyd A.T., Squires G.L., Fetters L.J., Rennie A.R., Horton J.C., de Vallèra A.M.B.G. Small-angle neutron scattering from star-branched polymers in dilute solution. // Macromolecules, 1989, vol. 22, No. 7, p. 3130-3137.

42. Hadjichristidis N., Roovers J.E.L. Synthesis and solution properties of linear, fourbranched and six-branched star polyisoprenes. // J. Polym. Sci., Polym. Phys. Ed., 1974, vol. 12, p. 2521-2533; Bauer B.J., Fetters L.J., Graessley W.W., Hadjichristidis N., Quack G.F. Chain dimensions in dilute polymer solutions: a light-scattering and viscometric study of multiarmed polyisoprene stars in good and $\Theta$ solvents. // Macromolecules, 1989, vol. 22, No. 5, p. 2337-2347.

43. Daoud M., Cotton J.P. Star shaped polymers: a model for the conformation and its concentration dependence. // J. Physique, 1982, vol. 43, No. 3, p. 531-538.

44. Murat M., Grest G.S. Molecular dynamics study of dendrimer molecules in solvents of varying quality. // Macromolecules, 1996, vol. 29, No. 4, p. 1278-1285.

45. Batoulis J., Kremer K. Residual 3-body interactions of a $\Theta$ polymer: star polymers. // Europhys Lett., 1988, vol. 7, No. 8, p. 683-688.

46. Bruns W., Carl W. Relations between averaged configurational properties of linear and starlike polymer models at the $\Theta$ temperature. // Macromolecules, 1991, vol. 24, No. 1, p. 209-212.

47. Khokhlov A.R. On the $\Theta$ behaviour of a polymer chain. // J. Physique, 1977, vol. 38, No. 7, p. 845-849; Concept of quasi-monomers and its application to some problems of polymer statistics. // Polymer, 1978, vol. 19, p. 1387-1396.

48. Forni A., Ganazzoli F., Vacatello M. Local conformation of regular star polymers in a good solvent: a Monte Carlo study. // Macromolecules, 1996, vol. 29, No. 8, p. 29942999.

49. Mansfield M.L. Dendron segregation in model dendrimers. // Polymer, 1994, vol. 35, No. 9, p. 1827-1830.

50. Zimm B.H., Kilb R.W. Dynamics of branched polymer molecules in dilute solution. // J. Pol. Sci., 1959, vol. 37, p. 19-42.

51. Cai C., Chen Z.Y. Rouse dynamics of a dendrimer model in the $\Theta$ condition. // Macromolecules, 1997, vol. 30, No. 17, p. 5104-5117.

52. Zimm B.H. Chain molecule hydrodynamics by the Monte Carlo method and the validity of the Kirkwood-Riseman approximation. // Macromolecules, 1980, vol. 13, No. 3, 
p. 592-602; Monte Carlo calculation of the friction coefficient and viscosity number of wormlike star molecules. // Macromolecules, 1984, vol. 17, No. 4, p. 795-798.

53. Lescanec R.L., Muthukumar M. Configurational characteristics and scaling behaviour of starburst molecules: a computational study. // Macromolecules, 1990, vol. 23, No. 8, p. 2280-2288.

54. Chen Z.Y., Cui S.-M. Monte Carlo simulations of star-burst dendrimers. // Macromolecules, 1996, vol. 29, No. 24, p. 7943-7952.

55. Ganazzoli F. Dynamics of regular star polymers: viscoelasticity and oscillatory flow birefringence. // J. Chem. Phys., 1997, vol. 106, No. 21, p. 8913-8917.

56. Rey A., Freire J.J. Dynamics of chains in excluded volume conditions: frequencydependent viscosity of regular star chains. // J. Chem. Phys., 1996, vol. 104, No. 2, p. $758-766$.

57. Richter D., Stühn B., Ewen B., Nerger D. Collective relaxation of star polymers a neutron spin echo study. // Phys. Rev. Lett., 1987, vol. 58, No. 23, p. 2462-2465; Richter D., Farago B., Fetters L.J., Huang J.S., Ewen B. On the relation between structure and dynamics of star polymers in solution. // Macromolecules, 1990, vol. 23, No. 6, p. 1845-1856.

58. Ganazzoli F. Linear, branched and hyperbranched macromolecules in dilute solution. // Macromol. Symp., 2002 (in press).

59. Allegra G., De Vitis M., Ganazzoli F. Chain collapse of star polymers. // Makromol. Chem., Theory Simul., 1993, vol. 2, p. 829-849.

60. Ganazzoli F., Kuznetsov Yu.A., Timoshenko E.G. Conformations of amphiphilic diblock star copolymers. // Macromol. Theory Simul., 2001, vol. 10, No. 4, p. 325-338.

61. Tsunashima Y. Experimental test of renormalization-group calculations on the universality of dilute-solution polymer dynamics. // Polymer, 1989, vol. 30, p. 2284-2287.

62. Ganazzoli F., Raffaini G. Non-Newtonian viscosity in linear and star polymers. // Macromol. Theory Simul., 1999, vol. 8, No. 3, p. 234-246. 


\section{Конформації та динаміка зірок і дендримерів: гаусівський самоузгоджений підхід}

\section{Ф.Ганаццолі}

Факультет хімії та хімічних технологій ім. Г.Натта

Міланського політехнічного інституту,

вул. Л.Манчінеллі 7, 20131 Мілан, Італія

Отримано 1 червня 2001 р.

Здійснено огляд деяких конформаційних та динамічних аспектів гілкових полімерів. Ми обговорюємо теоретичний гаусівський самоузгоджений підхід, запропонований у нашій групі, що використовується для вивчення поведінки регулярних зіркових полімерів та дендримерів в умовах різних розчинників. Ми розглядаємо в єдиних рамках як незбурений $\Theta$-стан, так і стан доброго розчинення, в порівнянні $з$ іншими теоретичними чи симуляційними підходами, а також з деякими експериментальними результатами. Ми коротко представляємо також подальші результати, отримані для амфіфільних кополімерних зірок у відбіркових розчинниках, з метою показати як можливості методу, так і його недоліки.

Ключові слова: зіркові полімери, дендримери, статистична механіка, конформації полімерів, динаміка полімерів.

PACS: 36.20.Ey, 61.25. Hq, 82.35.Jk, 82.35.Lr, 83.80.Rs 
